\title{
ON THE COLLOCATION METHODS FOR SINGULAR INTEGRAL EQUATIONS WITH HILBERT KERNEL
}

\author{
JINYUAN DU
}

\begin{abstract}
In the present paper, we introduce some singular integral operators, singular quadrature operators and discretization matrices of singular integral equations with Hilbert kernel. These results both improve the classical theory of singular integral equations and develop the theory of singular quadrature with Hilbert kernel. Then by using them a unified framework for various collocation methods of numerical solutions of singular integral equations with Hilbert kernel is given. Under the framework, it is very simple and obvious to obtain the coincidence theorem of collocation methods, then the existence and convergence for constructing approximate solutions are also given based on the coincidence theorem.
\end{abstract}

\section{INTRODUCTION}

Singular integral equations (SIEs) with Cauchy kernel often arise in mathematical models of physical phenomena. In the past thirty years, various collocation methods for SIEs with Cauchy kernel have been the topic of a great many of papers, most of which can be found in the two surveys [1, 2. D. Elliott successfully studied the more complete analytic theory for these collocation methods in a series of important papers 3-10. In 11, 12, Du systematically introduced some singular integral operators, singular quadrature operators and discretization matrices for singular integral equations with Cauchy kernel and gave a unified framework for various collocation methods. Singular integral equations with Hilbert kernel are also frequently encountered in physical and engineering applications. In this paper, we shall consider the collocation methods for singular integral equations with Hilbert kernel of the form

$$
a(t) \varphi(t)+\frac{b(t)}{2 \pi} \int_{0}^{2 \pi} \varphi(\tau) \cot \frac{\tau-t}{2} \mathrm{~d} \tau+\frac{\lambda}{2 \pi} \int_{0}^{2 \pi} k(\tau, t) \varphi(\tau) \mathrm{d} \tau=f(t), \quad 0 \leq t<2 \pi
$$

In (1.1), the input real-valued functions $a, b, f, k$ are Hölder-continuous functions with period $2 \pi$ for their arguments, denoted as $a, b, f, k \in H_{2 \pi}, \lambda$ is a given constant, and it is required to find the solution $\varphi$ in the class $H_{2 \pi}$ (see Chapter $\mathrm{V}$ in [13], Chapter I in [14]), the first integral is understood as the principle value integral

Received by the editor June 20, 2007 and, in revised form, May 11, 2008.

2000 Mathematics Subject Classification. Primary 65E05, 65J10, 41A55, 42A10.

Key words and phrases. Singular integral equations with Hilbert kernel, Hilbert singular integral operators, Hilbert singular quadrature operators, discretization matrices, collocation methods.

This project was supported by NNSF of China (\#10471107) and RFDP of Higher Eduction of China (\#20060486001).

(C)2008 American Mathematical Society Reverts to public domain 28 years from publication 
with Hilbert kernel and at $t=0$ (or $t=2 \pi)$ as

$$
\int_{0}^{2 \pi} \varphi(\tau) \cot \frac{\tau}{2} \mathrm{~d} \tau=\lim _{\delta \rightarrow 0^{+}} \int_{\delta}^{2 \pi-\delta} \varphi(\tau) \cot \frac{\tau}{2} \mathrm{~d} \tau .
$$

In 1978, S. Krenk discussed the numerical method for SIE (1.1) in the case that $a(t)$ and $b(t)$ are constants [15]. In 1983, for the same case, N. I. Ioakimidis rediscussed it by using a somewhat different method [16. He gave the result on the equivalence of the approximate solutions obtained by the direct and indirect quadrature methods via concrete calculations, which is called the coincidence theorem in the present paper. For general cases, in [17, 18, Du discussed the numerical methods for SIE (1.1), and the existence and convergence were demonstrated on the basis of the coincidence theorem which is established by applying again specific computation. In [19], J. Saranen and G. Vainikko thoroughly examined the more general framework of the collocation method with trigonometric trial functions and uniform grid. There, optimal convergence order in the scale of periodic Sobolev space is established, but only the case of index 0 is treated, a simple matrix form of the method. Then, J. Saranen and L. Schroderus extended some of the results in [19] to the scale of periodic Hölder spaces [20].

In the present paper, we shall give a unified framework for various collocation methods of SIE (1.1) and establish analytic theory for it. First of all we establish some necessary preliminaries including some special concepts and symbols in $\S 2-\S 4$. Some singular integral operators, singular quadrature operators and discretization matrices are introduced in $\S 5-\S 7$, which possess very interesting properties. These results improve both the classical theory of singular integral equations and the theory of singular quadrature with Hilbert kernel. Then, using these tools we give a unified framework for various collocation methods of SIE (1.1). The direct quadrature method of the framework is stated in $\S 8$, which is convenient for practical application. The indirect quadrature method of the framework is stated in $\S 9$, which is rather good for theoretical analysis. In $\S 10$, we verify the coincidence theorem of collocation methods that the approximate solutions obtained by the direct and indirect quadrature methods are completely the same, which is very simply and obviously obtained under the framework here. In $\S 11$ we establish the existence and convergence for the approximate solutions based on the coincidence theorem. In $\S 12$, some examples are given, which bring into being some earlier results.

\section{Normalized EQUATION}

In the first place, we must separate a weight function from the output function $\varphi$ in SIE (1.1). This step is very necessary, if not, the numerical method for SIE (1.1) will be only effective for the case that $a$ and $b$ are constants. To do so, we need some special concepts and symbols used throughout this paper.

Let

$$
\Theta(t)=\arg [a(t)-i b(t)],
$$

and take a continuous branch on $[0,2 \pi]$. Since $a(t)$ and $b(t)$ are real $2 \pi$-periodic functions, then the number

$$
\kappa=\frac{1}{2 \pi}[\Theta(2 \pi)-\Theta(0)]
$$


is an integer. We know that $2 \kappa$ is just the index of SIE (1.1) (see Chapter V in [13).

The mean value of $\Theta$ on $[0,2 \pi]$,

$$
\theta=\frac{1}{2 \pi} \int_{0}^{2 \pi} \Theta(\tau) \mathrm{d} \tau
$$

is called the characteristic number of SIE (1.1) which plays an important role thereinafter.

The canonical function $X(z)$ and the fundamental function $Z(t)$ of SIE (1.1) are, respectively, [13]

$$
\begin{aligned}
& X(z)=\left\{\begin{array}{l}
\exp \left\{\frac{1}{2 \pi} \int_{0}^{2 \pi}[\Theta(\tau)-\kappa \tau] \cot \frac{\tau-z}{2} \mathrm{~d} \tau+\kappa i z\right\}, \text { if } \operatorname{Im} z>0, \\
\exp \left\{\frac{1}{2 \pi} \int_{0}^{2 \pi}[\Theta(\tau)-\kappa \tau] \cot \frac{\tau-z}{2} \mathrm{~d} \tau-\kappa i z\right\}, \text { if } \operatorname{Im} z<0,
\end{array}\right. \\
& Z(t)=\exp \left\{\frac{1}{2 \pi} \int_{0}^{2 \pi}[\Theta(\tau)-\kappa \tau] \cot \frac{\tau-t}{2} \mathrm{~d} \tau\right\} \in H_{2 \pi}, \quad \text { if } \operatorname{Im} t=0 .
\end{aligned}
$$

We assume that SIE (1.1) is of the normal type, i.e.,

$$
r(t)=\sqrt{a^{2}(t)+b^{2}(t)} \neq 0 .
$$

The real-valued functions

$$
w_{1}(t)=\frac{Z(t)}{r(t)} \in H_{2 \pi}, \quad w_{2}(t)=\frac{1}{r(t) Z(t)} \in H_{2 \pi}
$$

are called, respectively, the weight functions of the first kind and the second kind associated with SIE (1.1).

Let

$$
\varphi(\tau)=w_{1}(\tau) y(\tau)
$$

then SIE (1.1) will become

$$
\begin{aligned}
& a(t) w_{1}(t) y(t)+\frac{b(t)}{2 \pi} \int_{0}^{2 \pi} w_{1}(\tau) y(\tau) \cot \frac{\tau-t}{2} \mathrm{~d} \tau \\
& +\frac{\lambda}{2 \pi} \int_{0}^{2 \pi} w_{1}(\tau) k(\tau, t) y(\tau) \mathrm{d} \tau=f(t), \quad 0 \leq t<2 \pi,
\end{aligned}
$$

which is called the normalized equation of SIE (1.1). Now we easily get the following result.

Theorem 2.1. With the relation (2.7), the solutions of SIE (1.1) in $H_{2 \pi}$ are equivalent to the solutions of SIE (2.8) in $\mathrm{H}_{2 \pi}$.

\section{QUASI-PRINCIPAL PART}

The mapping $w=e^{i z}$ maps, respectively, the upper strip region $S^{+}=\{z: \operatorname{Im} z>$ $0,0 \leq \operatorname{Re} z \leq 2 \pi\}$ and the lower strip region $S^{-}=\{z: \operatorname{Im} z<0,0 \leq \operatorname{Re} z \leq 2 \pi\}$ into the interior and exterior of the unit circle $\{w:|w|<1\}$ with the infinity accumulation point $z=+\infty i$ of $S^{+}$and the infinity accumulation point $z=-\infty i$ of $S^{-}$to $w=0$ and $w=\infty$, the straight lines $\operatorname{Re} z=0$ and $\operatorname{Re} z=2 \pi$ into the upper and lower banks of the cut $(0,+\infty)$. Let $\Phi^{+}(z)$ be a holomorphic function 
with period $2 \pi$ on the upper complex half-plane $\mathcal{C}^{+}=\{z: \operatorname{Im} z>0\}$, denoted by $\Phi^{+} \in \mathcal{A}_{2 \pi}^{+}$. Then $\left(\Phi^{+}\right)^{*}(w)=\Phi^{+}(z)$ with $w=e^{i z}$ is just well defined and analytic in $0<|w|<1$, so $\left(\Phi^{+}\right)^{*}$ has a Laurent expansion; thus we know easily that there is also the unique expansion in series form for $\Phi^{+}$[21]:

$$
\Phi^{+}(z)=\sum_{-\infty}^{+\infty} a_{j} e^{i j z}(\operatorname{Im} z>0) \quad \text { with } \quad a_{j}=\frac{1}{2 \pi} \int_{L} \Phi^{+}(z) e^{-i j z} \mathrm{~d} z,
$$

$$
\begin{gathered}
L:=\{z: z=x+i r, 0 \leq x \leq 2 \pi \text { with } r>0 \text { being an arbitrary constant }\} \\
\text { or } L:=\{z: z=x, 0 \leq x \leq 2 \pi\} \text { while } \Phi^{+} \text {is continuous to }[0,2 \pi] .
\end{gathered}
$$

We denote the whole complex plan by $\mathcal{C}$. Let

$$
\operatorname{P.P}\left[\Phi^{+}\right](z)=\frac{a_{0}}{2}+\sum_{j=1}^{+\infty} a_{-j} e^{-i j z}, \quad z \in \mathcal{C} .
$$

We call it the principal part of $\Phi^{+}$at $z=+\infty i$. Obviously, it is an entire function with period $2 \pi$, denoted by P.P $\left[\Phi^{+}\right] \in \mathcal{A}_{2 \pi}$, and

$$
\begin{gathered}
\operatorname{P.P}\left[\Phi^{+}\right](-\infty i)=\lim _{y \rightarrow-\infty, 0 \leq x \leq 2 \pi} \operatorname{P.P}\left[\Phi^{+}\right](z)=\frac{a_{0}}{2}, \\
\lim _{y \rightarrow-\infty, 0 \leq x \leq 2 \pi}\left[\Phi^{+}(z)-\operatorname{P.P}\left[\Phi^{+}\right](z)\right]=\frac{a_{0}}{2},
\end{gathered}
$$

where $x=\operatorname{Re} z$ and $y=\operatorname{Im} z$ which is always so thereinafter. If

$$
\lim _{y \rightarrow+\infty, 0 \leq x \leq 2 \pi} e^{i m z} \Phi^{+}(z)=A \neq 0,
$$

then we say that $\Phi^{+}$has the pole of order $m$ at $z=+\infty i$, which is just equivalent to that $\left(\Phi^{+}\right)^{*}$ has the pole of order $m$ at $w=0$. In this case, we get

$$
\operatorname{P.P}\left[\Phi^{+}\right](z)= \begin{cases}0, & \text { if } m<0, \\ \frac{a_{0}}{2}+\sum_{j=1}^{m} a_{-j} e^{-i j z} \quad\left(a_{-m}=A\right), & \text { if } m \geq 0 .\end{cases}
$$

If $\Phi^{-}(z)$ is a holomorphic function with period $2 \pi$ on the lower complex halfplane $\mathcal{C}^{-}=\{z: \operatorname{Im} z<0\}$, denoted by $\Phi^{-} \in \mathcal{A}_{2 \pi}^{-}$, similarly, it also has the unique expansion in the series form:

$$
\begin{aligned}
& \Phi^{-}(z)=\sum_{-\infty}^{+\infty} b_{j} e^{i j z}(\operatorname{Im} z<0) \quad \text { with } \quad b_{j}=\frac{1}{2 \pi} \int_{\Gamma} \Phi^{-}(z) e^{-i j z} \mathrm{~d} z, \\
& \Gamma:=\{z: z=x-i r, 0 \leq x \leq 2 \pi \text { with } r>0 \text { being an arbitrary constant }\} \\
& \text { or } \Gamma:=\{z: z=x, 0 \leq x \leq 2 \pi\} \text { while } \Phi^{-} \text {is continuous to }[0,2 \pi] .
\end{aligned}
$$

We call

$$
\text { P.P }\left[\Phi^{-}\right](z)=\frac{b_{0}}{2}+\sum_{j=1}^{+\infty} b_{j} e^{i j z}, \quad z \in \mathcal{C}
$$

the principal part of $\Phi^{-}(z)$ at $z=-\infty i$. Obviously, P.P $\left[\Phi^{-}\right] \in \mathcal{A}_{2 \pi}$ and

$$
\begin{gathered}
\text { P.P }\left[\Phi^{-}\right](+\infty i)=\lim _{y \rightarrow+\infty, 0 \leq x \leq 2 \pi} \operatorname{P.P}\left[\Phi^{-}\right](z)=\frac{b_{0}}{2}, \\
\lim _{y \rightarrow+\infty, 0 \leq x \leq 2 \pi}\left[\Phi^{-}(z)-\operatorname{P.P}\left[\Phi^{-}\right](z)\right]=\frac{b_{0}}{2} .
\end{gathered}
$$


Similary, if

$$
\lim _{y \rightarrow-\infty, 0 \leq x \leq 2 \pi} e^{-i m z} \Phi^{-}(z)=B \neq 0,
$$

then we say that $\Phi^{-}$has the pole of order $m$ at $z=-\infty i$. In this case, we get

$$
\text { P.P }\left[\Phi^{-}\right](z)= \begin{cases}0, & \text { if } m<0, \\ \frac{b_{0}}{2}+\sum_{j=1}^{m} b_{j} e^{i j z}\left(b_{m}=B\right), & \text { if } m \geq 0 .\end{cases}
$$

If $\Phi(z)$ with period $2 \pi$ is a sectionally holomorphic function with the $x$-axis as its jump curve, i.e., $\left.\Phi\right|_{\mathcal{C}^{+}} \in A_{2 \pi}^{+}$and $\left.\Phi\right|_{\mathcal{C}^{-}} \in A_{2 \pi}^{-}$and there exist the boundary value functions (see Chapter II in [13])

$$
\Phi^{+}(\tau)=\lim _{z \rightarrow \tau, \operatorname{Im} z>0, \tau \in \mathcal{R}} \Phi(z) \in H_{2 \pi}, \quad \Phi^{-}(\tau)=\lim _{z \rightarrow \tau, \operatorname{Im} z<0, \tau \in \mathcal{R}} \Phi(z) \in H_{2 \pi}
$$

where $\mathcal{R}$ denotes the set of all real numbers, then we denote it by $\Phi \in \mathcal{A} H_{2 \pi}$. By (3.1) and (3.6) we have the following result.

Lemma 3.1. If $\Phi \in A H_{2 \pi}$, then $a_{-j} e^{-i j z}+b_{j} e^{i j z}=\alpha_{j} \sin j z+\beta_{j} \cos j z$ with

$$
\begin{aligned}
& \alpha_{j}=\frac{1}{2 \pi} \int_{0}^{2 \pi}\left[\left(\Phi^{+}(\tau)+\Phi^{-}(\tau)\right) \cos j \tau+i\left(\Phi^{+}(\tau)-\Phi^{-}(\tau)\right) \sin j \tau\right] \mathrm{d} \tau, \\
& \beta_{j}=\frac{1}{2 \pi} \int_{0}^{2 \pi}\left[\left(\Phi^{+}(\tau)+\Phi^{-}(\tau)\right) \sin j \tau+i\left(\Phi^{-}(\tau)-\Phi^{-}(\tau)\right) \cos j \tau\right] \mathrm{d} \tau .
\end{aligned}
$$

Let

$$
\text { Q.P }[\Phi](z)=\text { P.P }\left[\Phi^{+}\right](z)+\operatorname{P.P}\left[\Phi^{-}\right](z), \quad z \in \mathcal{C},
$$

which is called the quasi-principal part of $\Phi$. If

$$
\triangle(\Phi)=\frac{a_{0}-b_{0}}{2}, \triangle[\Phi](z)=\Phi(z)-\text { Q.P }[\Phi](z) \quad\left(\in \mathcal{A} H_{2 \pi}\right),
$$

they are respectively called the deficient number and deficient function of $\Phi$.

\section{Lemma 3.2.}

$$
\triangle(\Phi)=\frac{1}{4 \pi} \int_{0}^{2 \pi}\left[\Phi^{+}(\tau)-\Phi^{-}(\tau)\right] \mathrm{d} \tau=\triangle[\Phi](+\infty i)=-\triangle[\Phi](-\infty i) .
$$

Proof. These results follow from (3.1), (3.3), (3.6), (3.8) and (3.13).

Lemma 3.3. If $\Phi \in A H_{2 \pi}$, then

$$
\frac{1}{2 \pi} \int_{0}^{2 \pi}\left[\Phi^{+}(\tau)-\Phi^{-}(\tau)\right] \cot \frac{\tau-t}{2} \mathrm{~d} \tau=\Phi^{+}(t)+\Phi^{-}(t)-2 \mathrm{Q} . \mathrm{P}[\Phi](t) .
$$

Proof. Let $\varphi(\tau)=\Phi^{+}(\tau)-\Phi^{-}(\tau)(\operatorname{Im} \tau=0)$, we consider the jump problem (see Chapter II in [13])

$$
\varphi(\tau)=\Psi^{+}(\tau)-\Psi^{-}(\tau), \quad \operatorname{Im} \tau=0 .
$$

It is evident that $\triangle[\Phi]$ is a solution of the jump problem (3.14) and $\triangle[\Phi](+\infty i)=$ $-\triangle[\Phi](-\infty i)=\triangle(\Phi)$ by Lemma 3.2. On the other hand, let

$$
(\mathbf{H}[\varphi])(z)=\frac{1}{4 \pi i} \int_{0}^{2 \pi} \varphi(\tau) \cot \frac{\tau-z}{2} \mathrm{~d} \tau, \quad \operatorname{Im} z \neq 0,
$$


then the Plemelj formula holds for any $\varphi \in H_{2 \pi}$ (see [22, 23, Chapter I in [13])

$$
\left\{\begin{array}{l}
(\mathbf{H}[\varphi])^{+}(t)=\frac{1}{2} \varphi(t)+\frac{1}{4 \pi i} \int_{0}^{2 \pi} \varphi(\tau) \cot \frac{\tau-t}{2} \mathrm{~d} \tau, \\
(\mathbf{H}[\varphi])^{-}(t)=-\frac{1}{2} \varphi(t)+\frac{1}{4 \pi i} \int_{0}^{2 \pi} \varphi(\tau) \cot \frac{\tau-t}{2} \mathrm{~d} \tau
\end{array} \quad t \in \mathcal{R} .\right.
$$

Thus $(\mathbf{H}[\varphi])$ is also a solution of the jump problem (3.14) and $(\mathbf{H}[\varphi])(+\infty i)=$ $-(\mathbf{H}[\varphi])(-\infty i)=\triangle(\Phi)$ by using Lemma 3.2. Thus, $\delta(z)=(\mathbf{H}[\varphi])(z)-\triangle[\Phi](z) \in$ $A_{2 \pi}$ and $\delta( \pm \infty i)=0$, so $\delta(z) \equiv 0$. This is to say that $\Phi(z)=(\mathbf{H}[\varphi])(z)+\mathrm{Q} . \mathrm{P}[\Phi](z)$; so, again by the Plemelj formula (3.16), the proof is completed.

\section{Trigonometric And PARATrigonometric POlYNOMials}

Let $H_{n}^{T}$ denote the class of all trigonometric polynomials with real coefficients of degree not greater than $n$ and regard $H_{n}^{T}=\{0\}$ if $n<0$, and let $H_{n}^{T *}$ denote the class of all trigonometric polynomials of degree $n(n \geq 0)$. Let $H_{n}^{T}(\alpha)$ denote the family of trigonometric polynomials of the form

$$
f(t)=c_{n} \sin (n t+\alpha)+T_{n-1}(t), \quad T_{n-1} \in H_{n-1}^{T}, \quad 0 \leq \alpha<\pi, n \geq 1 .
$$

$c_{n}$ is called the coefficient of the highest term. Clearly, $f$ is of degree $n$ if and only if $c_{n} \neq 0(n>0)$. $H_{n}^{T *}(\alpha)$ denotes the family of trigonometric polynomials of degree $n$ in $H_{n}^{T}(\alpha)(n>0)$. Obviously, $H_{0}^{T}(0)=\{0\}$ and $H_{0}^{T}(\alpha)=\mathcal{R}(\alpha \neq 0)$, $H_{n}^{T *}\left(\alpha_{1}\right) \cap H_{n}^{T *}\left(\alpha_{2}\right)=\emptyset$ while $\alpha_{1} \neq \alpha_{2}$ and $n>0$. Moreover, we regard $H_{0}^{T *}(\alpha)$ with any $\alpha$ as the set of all nonzero reals and $H_{n}^{T *}=H_{n}^{T}(\alpha)=H_{n}^{T *}(\alpha)=\{0\}$ if $n<0$.

If $f$ is Hölder-continuous and $f(t+2 \pi)=-f(t)$, we say that it is $2 \pi$-antiperiodic, denoted as $f \in \bar{H}_{2 \pi}$. If

$$
f(t)=\sum_{j=0}^{n}\left[a_{j} \sin \left(j+\frac{1}{2}\right) t+b_{j} \cos \left(j+\frac{1}{2}\right) t\right] \text { with } a_{n}^{2}+b_{n}^{2} \neq 0,
$$

then we call it a paratrigonometric polynomial of degree $\left(n+\frac{1}{2}\right)$ and write $f \in$ $H_{n+\frac{1}{2}}^{T *}$, where the coefficients $a_{j}$ 's and $b_{j}$ 's are real. Let $H_{n+\frac{1}{2}}^{T}$ denote the class of all paratrigonometric polynomials of degree not greater than $\left(n+\frac{1}{2}\right)$. Obviously, $H_{n+\frac{1}{2}}^{T} \subset \bar{H}_{2 \pi}$ and regard $H_{n+\frac{1}{2}}^{T}=\{0\}$ if $n<0$. Let $H_{n+\frac{1}{2}}^{T}(\alpha)$ denote the family of all paratrigonometric polynomials of the form

$$
c_{n} \sin \left[\left(n+\frac{1}{2}\right) t+\alpha\right]+T_{n-\frac{1}{2}}(t), \quad T_{n-\frac{1}{2}} \in H_{n-\frac{1}{2}}^{T}, \quad 0 \leq \alpha<\pi, \quad n \geq 0 .
$$

$c_{n}$ is called the coefficient of the term of degree $\left(n+\frac{1}{2}\right)$. If $c_{n} \neq 0$, we denote it $\in H_{n+\frac{1}{2}}^{T *}(\alpha)$. Obviously, $H_{n+\frac{1}{2}}^{T *}\left(\alpha_{1}\right) \cap H_{n+\frac{1}{2}}^{T *}\left(\alpha_{2}\right)=\emptyset$ while $\alpha_{1} \neq \alpha_{2}$. We also regard $H_{n+\frac{1}{2}}^{T *}=H_{n+\frac{1}{2}}^{T *}(\alpha)=H_{n+\frac{1}{2}}^{T}(\alpha) \stackrel{2}{=}\{0\}$ while $n<0$.

Lemma 4.1 (see [24, 25]). If $F \in H_{\frac{1}{2} k}^{T *}(\alpha)(k>0), G \in H_{\frac{1}{2} r}^{T *}(\beta)(r>0)$, then $F G \in H_{\frac{1}{2}(k+r)}^{T *}\left([\pi / 2+\alpha+\beta]_{\pi}\right)$ where $[\vartheta]_{\pi}$ denotes the number congruent to $\vartheta(\bmod \pi)$ in $[0, \pi)$. 
Lemma 4.2 (see [25, 26]). If $f \in H_{n}^{T *}(\alpha)(n>0)$ with the highest coefficient $c_{n}, F(\tau, t)=[f(\tau)-f(t)] \cot \frac{\tau-t}{2}$, then $F(\tau, t)=c_{n}[\cos (n \tau+\alpha)+\cos (n t+\alpha)]+$ $\sum_{j=1}^{n-1}\left[A_{j}(t) \sin (n-j) \tau+B_{j}(t) \cos (n-j) \tau\right]$ where $A_{j}, B_{j} \in H_{j}^{T *}$.

Lemma 4.3 (Euclidean division formula). If $G_{\frac{1}{2} \ell}^{\beta} \in H_{\frac{1}{2} \ell}^{T *}(\beta)(\ell>0)$ and $F_{\frac{1}{2} j} \in H_{\frac{1}{2} j}^{T}$, then there uniquely exist $Q_{\frac{1}{2}(j-\ell)} \in H_{\frac{1}{2}(j-\ell)}^{T}$ and $R$ such that $F_{\frac{1}{2} j}=G_{\frac{1}{2} \ell}^{\beta} Q_{\frac{1}{2}(j-\ell)}+R$, where $R \in H_{\frac{1}{2} \ell}^{T}\left([\pi / 2+\beta]_{\pi}\right)$ when $(j-\ell)$ is even and $R \in H_{\frac{1}{2}(\ell-1)}^{T}$ when $(j-\ell)$ is odd.

Proof. The uniqueness is clear. For the existence, if $j \leq \ell$, then the conclusion is also obvious. If $j>\ell$, there is $q_{\frac{1}{2}(j-\ell)} \in H_{\frac{1}{2}(j-\ell)}^{T}\left([\pi / 2+\alpha-\beta]_{\pi}\right)$ such that $F_{\frac{1}{2} j}-$ $G_{\frac{1}{2} \ell}^{\beta} q_{\frac{1}{2}(j-\ell)} \in H_{\frac{1}{2}(j-1)}^{T}$ by Lemma 4.1, then using the inductive method the proof is completed.

Lemma 4.4 (Factorization). If $F_{\frac{1}{2} j} \in H_{\frac{1}{2} j}^{T *}(j>0)$ and $F_{\frac{1}{2} j}\left(t_{0}\right)=0$, then $F_{\frac{1}{2} j}(t)=$ $\sin \frac{1}{2}\left(t-t_{0}\right) Q_{\frac{1}{2}(j-1)}(t)$ where $Q_{\frac{1}{2}(j-1)} \in H_{\frac{1}{2}(j-1)}^{T *}$.

Proof. Taking $G_{\frac{1}{2} \ell}^{\beta}(t)=\sin \frac{1}{2}\left(t-t_{0}\right)$ in Lemma 4.3 , the proof is immediately completed.

Example 4.1. This example is used frequently thereinafter. Let $X$ be the canonical function of SIE (1.1) and $\theta$ the characteristic number of SIE (1.1). By the Plemelj formula we know

$$
\left\{\begin{array}{l}
X^{+}(\tau)-X^{-}(\tau)=-2 i b(\tau) w_{1}(\tau) \\
X^{+}(\tau)+X^{-}(\tau)=2 a(\tau) w_{1}(\tau) \\
\left(X^{-1}\right)^{+}(\tau)-\left(X^{-1}\right)^{-}(\tau)=-2 i b(\tau) w_{2}(\tau) \\
\left(X^{-1}\right)^{+}(\tau)+\left(X^{-1}\right)^{-}(\tau)=2 a(\tau) w_{2}(\tau)
\end{array}\right.
$$

This implies that $\alpha_{j}$ and $\beta_{j}$ in Lemma 3.1 are real, while $\Phi=X$. Moreover, noting that

$$
\lim _{y \rightarrow+\infty, x \in \mathcal{R}}\left[e^{-i \kappa z} X(z)\right]=(-1)^{\kappa} e^{i \theta}, \lim _{y \rightarrow-\infty, x \in \mathcal{R}}\left[e^{i \kappa z} X(z)\right]=(-1)^{\kappa} e^{i \theta} .
$$

Now we get, by (3.5),(3.10), (4.4) and Lemma 3.2,

$$
\begin{array}{cl}
\text { Q.P }[X] \in H_{-\kappa}^{T *}\left([\pi / 2-\theta]_{\pi}\right), & \text { Q.P }\left[X^{-1}\right] \in H_{\kappa}^{T *}\left([\pi / 2+\theta]_{\pi}\right), \\
\triangle(X)=\frac{1}{2 \pi i} \int_{0}^{2 \pi} b(\tau) w_{1}(\tau) \mathrm{d} \tau, & \triangle\left(X^{-1}\right)=\frac{1}{2 \pi i} \int_{0}^{2 \pi} b(\tau) w_{2}(\tau) \mathrm{d} \tau,
\end{array}
$$

in particular,

$$
\begin{aligned}
\text { Q.P }[X](\tau)=\text { Q.P }\left[X^{-1}\right](\tau)= & \cos \theta, \quad \triangle(X)=-\triangle\left(X^{-1}\right)=i \sin \theta, \\
& \text { if } \kappa=0,
\end{aligned}
$$

In general, we may get, in the same way,

$$
\begin{gathered}
\text { Q.P }\left[X T_{r}^{\alpha}\right] \in H_{r-\kappa}^{T *}\left([\alpha-\theta]_{\pi}\right), \quad \text { Q.P }\left[X^{-1} T_{r}^{\alpha}\right] \in H_{r+\kappa}^{T *}\left([\alpha+\theta]_{\pi}\right), \\
\text { for } T_{r} \in H_{r}^{T *}(\alpha)(r>0) .
\end{gathered}
$$




\section{Singular integral operators}

We introduce the singular integral operators (SIOs)

$$
\begin{aligned}
& (\mathbf{A} y)(t)=a(t) w_{1}(t) y(t)+\frac{b(t)}{2 \pi} \int_{0}^{2 \pi} w_{1}(\tau) y(\tau) \cot \frac{\tau-t}{2} d \tau, \\
& (\mathbf{B} y)(t)=a(t) w_{2}(t) y(t)-\frac{b(t)}{2 \pi} \int_{0}^{2 \pi} w_{2}(\tau) y(\tau) \cot \frac{\tau-t}{2} d \tau
\end{aligned}
$$

and their adjoint operators

$$
\begin{aligned}
& \left(\mathbf{A}^{*} y\right)(t)=a(t) w_{1}(t) y(t)+\frac{1}{2 \pi} \int_{0}^{2 \pi} w_{1}(\tau) b(\tau) y(\tau) \cot \frac{\tau-t}{2} d \tau, \\
& \left(\mathbf{B}^{*} y\right)(t)=a(t) w_{2}(t) y(t)-\frac{1}{2 \pi} \int_{0}^{2 \pi} w_{2}(\tau) b(\tau) y(\tau) \cot \frac{\tau-t}{2} d \tau .
\end{aligned}
$$

Remark 5.1. For convenience thereinafter, here we denote the adjoint operator of $\mathbf{A}$ as $\mathbf{B}^{*}$, of $\mathbf{B}$ as $\mathbf{A}^{*}$, but as $\mathbf{A}^{\prime}$ and $\mathbf{B}^{\prime}$ in general texts (see Chapter $\mathrm{V}$ in [13, Chapter I in [14]).

Remark 5.2. A, $\mathbf{B}, \mathbf{A}^{*}, \mathbf{B}^{*}$ all are SIOs from $H_{2 \pi}$ to $H_{2 \pi}$, i.e., $\mathbf{A}, \mathbf{B}, \mathbf{A}^{*}, \mathbf{B}^{*}: H_{2 \pi} \rightarrow$ $H_{2 \pi}$.

Theorem 5.1. Let $T \in \mathcal{A} H_{2 \pi}$, then $\left(\mathbf{A}^{*} T\right)(\tau)=\mathrm{Q} \cdot \mathrm{P}[X T](\tau), \quad\left(\mathbf{B}^{*} T\right)(\tau)=$ Q.P $\left[X^{-1} T\right](\tau)$.

Proof. Taking, respectively, $\Phi=X T$ and $\Phi=X^{-1} T$ in Lemma 3.3 and using (4.4), the proof follows.

Again, we introduce the integral operators

$$
\begin{aligned}
& \left(\mathbf{D}_{1} y\right)(t)=\frac{1}{2 \pi} \int_{0}^{2 \pi} w_{1}(\tau) d_{1}(\tau, t) y(\tau) \mathrm{d} \tau \\
& \left(\mathbf{D}_{2} y\right)(t)=\frac{1}{2 \pi} \int_{0}^{2 \pi} w_{2}(\tau) d_{2}(\tau, t) y(\tau) \mathrm{d} \tau
\end{aligned}
$$

where

$$
\begin{gathered}
d_{1}(\tau, t)=\left\{\mathrm{Q} . \mathrm{P}\left[X^{-1}\right](\tau)-\mathrm{Q} . \mathrm{P}\left[X^{-1}\right](t)\right\} \cot \frac{\tau-t}{2}-i \triangle\left(X^{-1}\right), \\
d_{2}(\tau, t)=\{\mathrm{Q} . \mathrm{P}[X](\tau)-\mathrm{Q} \cdot \mathrm{P}[X](t)\} \cot \frac{\tau-t}{2}-i \triangle(X) .
\end{gathered}
$$

Remark 5.3. By (4.6) and (4.7) in Example 4.1 and quoting Lemma 4.2, we easily see that

$$
d_{1}(\cdot, t) \in H_{\kappa}^{T}\left([\theta]_{\pi}\right), \quad d_{2}(\cdot, t) \in H_{-\kappa}^{T}\left([-\theta]_{\pi}\right)
$$


writing $B_{1, \kappa}(\tau)=2(-1)^{\kappa+1} \sin (\kappa \tau+\theta)$ and $B_{2,-\kappa}(\tau)=2(-1)^{\kappa+1} \sin (-\kappa \tau-\theta)$, then

$$
\begin{gathered}
d_{1}(\tau, t)=B_{1, \kappa}(\tau)+\sum_{j=1}^{\kappa-1}\left[A_{1, j}(t) \sin (\kappa-j) \tau+B_{1, j}(t) \cos (\kappa-j) \tau\right] \\
+\left[B_{1, \kappa}(t)-i \triangle\left(X^{-1}\right)\right] \quad \text { if } \quad \kappa>0, \\
d_{2}(\tau, t)=B_{1, \kappa}(\tau)+\sum_{j=1}^{\kappa-1}\left[A_{2, j}(t) \sin (\kappa-j) \tau+B_{2, j}(t) \cos (\kappa-j) \tau\right] \\
+\left[B_{2, \kappa}(t)-i \triangle\left(X^{-1}\right)\right] \quad \text { if } \quad \kappa<0, \\
d_{1}(\tau, t)=-i \triangle(X)=-\sin \theta, \quad d_{2}(\tau, t)=-i \triangle\left(X^{-1}\right)=\sin \theta \quad \text { if } \quad \kappa=0,
\end{gathered}
$$

where $A_{1, j}, B_{1, j}, A_{2, j}, B_{2, j}$ are some trigonometric polynomials of degree $j$.

Theorem 5.2. $\quad \mathbf{A B}=\mathbf{I}+b \mathbf{D}_{2}, \mathbf{B A}=\mathbf{I}-b \mathbf{D}_{1}$, where $\mathbf{I}$ is the identity operator.

Proof. We only prove the first equality since the other is similar. By Remark 5.2 the compositions of $\mathbf{A}$ and $\mathbf{B}$ are well defined. By the Poincaré-Bertrand formula (see Chapter I in [13] or [14]), Theorem 5.1, (5.3), (4.6) and the equality

$$
\cot \alpha \cot \beta=\cot (\beta-\alpha)[\cot \alpha-\cot \beta]-1,
$$

we get

$$
\begin{aligned}
& (\mathbf{A B} y)(x)=a(x) w_{1}(x)(\mathbf{B})(x)+\frac{b(x)}{2 \pi} \int_{0}^{2 \pi} w_{1}(\tau) a(\tau) w_{2}(\tau) y(\tau) \cot \frac{\tau-x}{2} \mathrm{~d} \tau \\
& +\frac{b^{2}(x)}{r^{2}(x)} y(x)+\frac{b(x)}{2 \pi} \int_{0}^{2 \pi} w_{2}(\tau) y(\tau)\left[\frac{1}{2 \pi} \int_{0}^{2 \pi} w_{1}(t) b(t) \cot \frac{t-\tau}{2} \cot \frac{t-x}{2} \mathrm{~d} t\right] \mathrm{d} \tau \\
& =y(x)+\frac{b(x)}{2 \pi} \int_{0}^{2 \pi} w_{2}(\tau) y(\tau)\left\{\left[\left(\mathbf{A}^{*} 1\right)(\tau)-\left(\mathbf{A}^{*} 1\right)(x)\right] \cot \frac{\tau-x}{2}-i \triangle(X)\right\} \mathrm{d} \tau \\
& =y(x)+\frac{b(x)}{2 \pi} \int_{0}^{2 \pi} w_{2}(\tau) y(\tau)\left\{[\mathrm{Q} \cdot \mathrm{P}[X](\tau)-\mathrm{Q} \cdot \mathrm{P}[X](x)] \cot \frac{\tau-x}{2}-i \triangle(X)\right\} \mathrm{d} \tau .
\end{aligned}
$$

Remark 5.4. From (5.6) in Remark 5.3 we know $\mathbf{D}_{1}: H_{2 \pi} \rightarrow H_{\kappa}^{T}\left([\theta]_{\pi}\right)$ and $\mathbf{D}_{2}$ : $H_{2 \pi} \rightarrow H_{-\kappa}^{T}\left([-\theta]_{\pi}\right)$. In particular, if $\kappa>0$, then $\mathbf{D}_{2}=\mathbf{0}$, this is to say that $\mathbf{A}$ has the right inverse $\mathbf{B}$ (but no left inverse). If $\kappa<0$, then $\mathbf{D}_{1}=\mathbf{0}$, this is to say that $\mathbf{A}$ has the left inverse $\mathbf{B}$ (but no right inverse). If $\kappa=0$, then

$$
\left(\mathbf{D}_{1} y\right)(t)=-\frac{\sin \theta}{2 \pi} \int_{0}^{2 \pi} w_{1}(\tau) y(\tau) \mathrm{d} \tau \text { and }\left(\mathbf{D}_{2} y\right)(t)=\frac{\sin \theta}{2 \pi} \int_{0}^{2 \pi} w_{2}(\tau) y(\tau) \mathrm{d} \tau .
$$

Corollary 5.1. Ima $\left(\mathbf{D}_{1}\right)=H_{\kappa}^{T}\left([\theta]_{\pi}\right)$ and $\operatorname{Ima}\left(\mathbf{D}_{2}\right)=H_{-\kappa}^{T}\left([-\theta]_{\pi}\right)$. If $\kappa \neq 0$ or $\kappa=0$ with $[\theta]_{\pi}=\pi / 2$, then $\operatorname{ker}(\mathbf{A})=b H_{\kappa}^{T}\left([\theta]_{\pi}\right), \operatorname{ker}(\mathbf{B})=b H_{-\kappa}^{T}\left([-\theta]_{\pi}\right), \mathbf{D}_{1} b T_{\kappa}^{\theta}=$ $T_{\kappa}^{\theta}, \mathbf{D}_{2} b T_{-\kappa}^{-\theta}=-T_{-\kappa}^{-\theta}$ where $T_{\kappa}^{\theta} \in H_{\kappa}^{T}\left([\theta]_{\pi}\right)$ and $T_{-\kappa}^{-\theta} \in H_{-\kappa}^{T}\left([-\theta]_{\pi}\right)$. If $\kappa=0$ with $[\theta]_{\pi} \neq \pi / 2$, then $\operatorname{ker}(\mathbf{A})=0$ and $\operatorname{ker}(\mathbf{B})=\{0\}$. The symbols Ima $(\cdot)$ and $\operatorname{ker}(\cdot)$ denote, respectively, the image set and the kernel space of an operator.

Proof. (I) By Remark 4.5 we have Ima $\left(\mathbf{D}_{1}\right) \subseteq H_{\kappa}^{T}\left([\theta]_{\pi}\right)$. (II) If $y \in \operatorname{ker}(\mathbf{A})$, then BA $y=0$ which implies $y=b \mathbf{D}_{1} y \in b H_{\kappa}^{T}\left([\theta]_{\pi}\right)$ by Theorem 5.2, i.e., $\operatorname{ker}(\mathbf{A}) \subseteq$ $b H_{\kappa}^{T}\left([\theta]_{\pi}\right)$. (III) If $\kappa \neq 0$ or $\kappa=0$ with $[\theta]_{\pi}=\pi / 2$, then $\mathbf{A}\left(b T_{\kappa}^{\theta}\right)=b \mathbf{A}^{*}\left(T_{\kappa}^{\theta}\right)=$ 
bQ.P $\left[X T_{\kappa}^{\theta}\right]=0$ for $T_{\kappa}^{\theta} \in H_{\kappa}^{T}(\theta)$ by Theorems 5.1 and 5.2 , (4.7) and (4.8), i.e., $b H_{\kappa}^{T}\left([\theta]_{\pi}\right) \subseteq \operatorname{ker}(\mathbf{A})$. In particular, we have $b T_{\kappa}^{\theta}=b \mathbf{D}_{1}\left(b T_{\kappa}^{\theta}\right)$ by $(\mathbb{I})$. Again noting $b \neq 0$ (does not vanish for some $t$ ) in the cases discussed now, hence $T_{\kappa}^{\theta}=\mathbf{D}_{1}\left(b T_{\kappa}^{\theta}\right)$, this is just $H_{\kappa}^{T}\left([\theta]_{\pi}\right) \subseteq \operatorname{Ima}\left(\mathbf{D}_{1}\right)$. (IV) For the case $\kappa=0$ with $[\theta]_{\pi} \neq \pi / 2$, Ima $\left(\mathbf{D}_{1}\right)=$ $H_{\kappa}^{T}\left([\theta]_{\pi}\right)$ is clear by Remark 5.4 and $\int_{0}^{2 \pi} w_{1}(\tau) \mathrm{d} \tau>0$. Moreover, if $\mathbf{A} y=0$, then $y=b \mathbf{D}_{1} y$ by (II), which implies $0=\mathbf{A}\left(b \mathbf{D}_{1} y\right)=b \mathbf{D}_{1} y \mathbf{A}^{*} 1=\cos \theta b \mathbf{D}_{1} y$ since $\cos \theta$ is a nonzero constant; finally $y=b \mathbf{D}_{1} y=0$, i.e., $\operatorname{ker}(\mathbf{A})=\{0\}$. Similarly, the assertions for $\mathbf{B}$ and $\mathbf{D}_{2}$ may be proved.

Corollary 5.2. If $\kappa \neq 0$ or $\kappa=0$ with $[\theta]_{\pi}=\pi / 2$, then $b \mathbf{D}_{1}$ and $-b \mathbf{D}_{2}$ are idempotent, $\mathbf{D}_{1}$ and $\mathbf{D}_{2}$ are left zero divisors of $\mathbf{B}$ and $\mathbf{A}$, respectively, $b \mathbf{D}_{1}$ and $b \mathbf{D}_{2}$ are right zero divisors of $\mathbf{A}$ and $\mathbf{B}$, respectively, i.e., $\mathbf{D}_{1} \mathbf{B}=0, \mathbf{D}_{2} \mathbf{A}=0$, $\mathbf{A} b \mathbf{D}_{1}=0$ and $\mathbf{B} b \mathbf{D}_{2}=0$.

Proof. If $\kappa \neq 0$ or $\kappa=0$ with $[\theta]_{\pi}=\pi / 2, \mathbf{A} b \mathbf{D}_{1}=0$ and $\mathbf{B} b \mathbf{D}_{2}=0$ are just the results of Corollary 5.1. Thus $(\mathbf{A B})^{2}=\mathbf{A}\left(\mathbf{I}-b \mathbf{D}_{1}\right) \mathbf{B}=\mathbf{A B}$, i.e., $\mathbf{A B}$ is idempotent, so is $b \mathbf{D}_{2}$. Noticing $\mathbf{B A B}=\mathbf{B}-b \mathbf{D}_{1} \mathbf{B}$ and $\mathbf{B} \mathbf{A B}=\mathbf{B}+\mathbf{B} b \mathbf{D}_{1}=\mathbf{B}$, thus $\mathbf{D}_{1} \mathbf{B}=0$. The rest of the proofs are obtained in the similar way.

Theorem 5.3. If $\kappa>0$ and $N_{\kappa} \in H_{\kappa}^{T}\left([\theta]_{\pi}\right)$ is a given trigonometric polynomial, then under the condition $\mathbf{D}_{1} y=N_{\kappa}, \mathbf{A} y=f$ possesses the unique solution $y=$ $\mathbf{B} f+b N_{\kappa}$. If $\kappa<0$, the condition of solvability for $\mathbf{A} y=f$ is $\mathbf{D}_{2} f=0$, and $\mathbf{A} y=f$ possesses the unique solution $y=\mathbf{B} f$ when the condition of solvability is fulfilled. If $\kappa=0$ with $[\theta]_{\pi}=\pi / 2$, the condition of solvability for $\mathbf{A} y=f$ is $\mathbf{D}_{2} f=0$, and under the condition $\mathbf{D}_{1} y=N$ where $N$ is a given constant, $\mathbf{A} y=f$ possesses the unique solution $y=\mathbf{B} f+b N$ while the condition of solvability is fulfilled. If $\kappa=0$ with $[\theta]_{\pi} \neq \pi / 2$, then $\mathbf{A} y=f$ possesses the unique solution

$$
y=\mathbf{B} f-b \sec \theta \mathbf{D}_{2} f=\mathbf{B} f-\frac{b}{2 \pi} \tan \theta \int_{0}^{2 \pi} w_{2}(\tau) f(\tau) d \tau .
$$

So we call $\mathbf{D}_{1}$ the unisolving operator and $\mathbf{D}_{2}$ the restricting operator.

Proof. When $\kappa>0$, obviously $y=\mathbf{B} f+b N_{\kappa}$ implies $\mathbf{A} y=f$ by Remark 5.4 and Corollary 5.1, as well as $\mathbf{D}_{1} y=N_{\kappa}$ by Corollaries 5.1-5.2, conversely, if $\mathbf{A} y=f$ and $\mathbf{D}_{1} y=N_{\kappa}$, then $\mathbf{B} f=(\mathbf{B A}) y=y-b \mathbf{D}_{1} y$ by Theorem 5.2, i.e., $y=\mathbf{B} f+b N_{\kappa}$. When $\kappa<0$, if $\mathbf{A} y=f$, then $\mathbf{D}_{2} f=0$ by Corollary 5.2, and $y=\mathbf{B} f$ by Remark 5.4, conversely, the latter two equations imply $\mathbf{A} y=f$ by Theorem 5.2. When $\kappa=0$ with $[\theta]_{\pi}=\pi / 2$, if $\mathbf{A} y=f$ with $\mathbf{D}_{1} y=N$, we then have that $\mathbf{D}_{2} f=\left(\mathbf{D}_{2} \mathbf{A}\right) y=0$ by Corollary 5.2 and $y=\mathbf{B} f+b N$ from $\mathbf{B A} y=\mathbf{B} f$ by Theorem 5.2 and Corollary 5.1, conversely while $\mathbf{D}_{2} f=0$ and $y=\mathbf{B} f+b N$, then $\mathbf{A} y=f$ by Theorem 5.2 and Corollary 5.1, as well as $\mathbf{D}_{1} y=N$ by Corollaries 5.1 and 5.2 . When $\kappa=0$ with $[\theta]_{\pi} \neq \pi / 2$, if $\mathbf{A} y=f$, then $y$ is unique from $\operatorname{ker}(\mathbf{A})=0$ in Corollary 5.1, and if $y=\mathbf{B} f-b \sec \theta \mathbf{D}_{2} f$ (its other form above may be obtained by Remark 5.4) then $\mathbf{A} y=\mathbf{A B} f-\mathbf{A}\left(b \sec \theta \mathbf{D}_{2} f\right)=f+b \mathbf{D}_{2} f-b \mathbf{D}_{2} f=f$.

Remark 5.5. From Theorem 5.3, we now know that $\mathbf{A}$ has neither a left inverse nor a right inverse if $\kappa=0$ with $[\theta]_{\pi}=\pi / 2$, and $\mathbf{A}$ has the inverse $\mathbf{A}^{-1}=\mathbf{B}-b \sec \theta \mathbf{D}_{2}$ if $\kappa=0$ with $[\theta]_{\pi} \neq \pi / 2$. 
Remark 5.6. When $\kappa<0$ or $\kappa=0$ with $[\theta]_{\pi}=\pi / 2, \mathbf{D}_{2} f=0$ is equivalent to $f \perp_{2} H_{-\kappa}^{T}\left([-\theta]_{\pi}\right)$, i.e.,

$$
\int_{0}^{2 \pi} w_{2}(\tau) h_{-\kappa}(\tau) f(\tau) d \tau=0 \text { for any } h_{-\kappa} \in H_{-\kappa}^{T}\left([-\theta]_{\pi}\right),
$$

which is clear from (5.6) in Remark 5.3.

Remark 5.7. Obviously, for SIE $\mathbf{B} y=f$ we also have results similar to those for SIE $\mathbf{A} y=f$ discussed above. For example, its condition of solvability when $\kappa>0$ or $\kappa=0$ with $[\theta]_{\pi}=\pi / 2$ is

$$
\int_{0}^{2 \pi} w_{1}(\tau) h_{\kappa}(\tau) f(\tau) \mathrm{d} \tau=0 \quad \text { for any } h_{\kappa} \in H_{\kappa}^{T}\left([\theta]_{\pi}\right),
$$

which is denoted by $f \perp_{1} H_{\kappa}^{T}\left([\theta]_{\pi}\right)$. When $\kappa=0$ with $[\theta]_{\pi} \neq \pi / 2$, then $\mathbf{B} y=f$ possesses a unique solution $y=\mathbf{A} f-b \sec \theta \mathbf{D}_{1} f$, or $y=\mathbf{A} f-\frac{b}{2 \pi} \tan \theta \int_{0}^{2 \pi} w_{1}(\tau) f(\tau) d \tau$.

Thereinafter we assume always that $b$ in SIE (2.8) is a trigonometric polynomial of degree $\mu$.

Theorem 5.4. If $T_{r} \in H_{r}^{T *}(\alpha)(r>0)$, then $\mathbf{A}\left(T_{r}\right) \in H_{r-\kappa}^{T *}\left([\alpha-\theta]_{\pi}\right)$ for $r>\kappa+\mu$ and $\mathbf{B}\left(T_{r}\right) \in H_{r+\kappa}^{T *}\left([\alpha+\theta]_{\pi}\right)$ for $r>\mu-\kappa$.

Proof. The first result is clear from $\left(\mathbf{A} T_{r}\right)(t)=$ Q.P $\left[X T_{r}\right](t)-\frac{1}{2 \pi} \int_{-1}^{1} w_{1}(\tau)[b(\tau)-$ $b(t)] T_{r}(\tau) \cot \frac{\tau-t}{2} \mathrm{~d} \tau$ and (4.8) in Example 4.1. The second result is similarly proved.

We will need a kind of singular integral operators with cosecant kernel. For $\bar{y} \in \bar{H}_{2 \pi}$, we introduce

$$
\begin{aligned}
& (\overline{\mathbf{A}} \bar{y})(t)=a(t) w_{1}(t) \bar{y}(t)+\frac{b(t)}{2 \pi} \int_{0}^{2 \pi} w_{1}(\tau) \bar{y}(\tau) \csc \frac{\tau-t}{2} d \tau \\
& (\overline{\mathbf{B}} \bar{y})(t)=a(t) w_{2}(t) \bar{y}(t)-\frac{b(t)}{2 \pi} \int_{0}^{2 \pi} w_{2}(\tau) \bar{y}(\tau) \csc \frac{\tau-t}{2} d \tau
\end{aligned}
$$

The singular integral with cosecant kernel for $\bar{y} \in \bar{H}_{2 \pi}$ is written as

$$
\begin{aligned}
\int_{0}^{2 \pi} \bar{y}(\tau) \csc \frac{\tau-t}{2} \mathrm{~d} \tau= & \int_{0}^{2 \pi} \bar{y}(\tau) \sin \frac{\tau-t}{2} \mathrm{~d} \tau \\
& +\int_{0}^{2 \pi} \bar{y}(\tau) \cos \frac{\tau-t}{2} \cot \frac{\tau-t}{2} \mathrm{~d} \tau
\end{aligned}
$$

where the first integral is a proper integral and the second integral is a singular integral with Hilbert kernel. Obviously, $\overline{\mathbf{A}}$ and $\overline{\mathbf{B}}$ map $\bar{H}_{2 \pi}$ into $\bar{H}_{2 \pi}$ and possess properties very similar to those of the SIOs $\mathbf{A}$ and $\mathbf{B}$. For example, in a way similar to Theorem 5.2, only substituting the equality (5.7) by the equality

$$
\csc \alpha \csc \beta=\csc (\beta-\alpha)[\cot \alpha-\cot \beta],
$$

we can get the following result. 
Theorem 5.5. $\quad \overline{\mathbf{A}} \overline{\mathbf{B}}=\mathbf{I}+b \overline{\mathbf{D}}_{2}, \quad \overline{\mathbf{B}} \overline{\mathbf{A}}=\mathbf{I}-b \overline{\mathbf{D}}_{1}$ where

$$
\begin{gathered}
\left(\overline{\mathbf{D}}_{1} \bar{y}\right)(t)=\frac{1}{2 \pi} \int_{0}^{2 \pi} w_{1}(\tau) \bar{y}(\tau)\left\{\text { Q.P }\left[X^{-1}\right](\tau)-\text { Q.P }\left[X^{-1}\right](t)\right\} \csc \frac{\tau-t}{2} \mathrm{~d} \tau, \\
\left(\overline{\mathbf{D}}_{2} \bar{y}\right)(t)=\frac{1}{2 \pi} \int_{0}^{2 \pi} w_{2}(\tau) \bar{y}(\tau)\{\text { Q.P }[X](\tau)-\text { Q.P }[X](t)\} \csc \frac{\tau-t}{2} \mathrm{~d} \tau .
\end{gathered}
$$

Remark 5.8. The conditions of solvability of $\bar{B} \bar{y}=\bar{f}$ and $\bar{A} \bar{y}=\bar{f}$ are, respectively,

$$
\begin{aligned}
& \overline{\mathbf{D}}_{1} \bar{f}=0 \quad \text { or } \quad \int_{0}^{2 \pi} w_{1}(\tau) \bar{h}_{\kappa-\frac{1}{2}} \bar{f}(\tau) \mathrm{d} \tau=0 \text { for any } \bar{h}_{\kappa-\frac{1}{2}} \in H_{\kappa-\frac{1}{2}}^{T}, \\
& \overline{\mathbf{D}}_{2} \bar{f}=0 \quad \text { or } \quad \int_{0}^{2 \pi} w_{2}(\tau) \bar{h}_{-\kappa-\frac{1}{2}} \bar{f}(\tau) \mathrm{d} \tau=0 \text { for any } \bar{h}_{-\kappa-\frac{1}{2}} \in H_{-\kappa-\frac{1}{2}}^{T} .
\end{aligned}
$$

\section{Singular quadrature operators}

In this section, we construct some singular quadrature operators (SQOs) which possess some properties similar to those of SIO A and $\mathbf{B}$ in the last section.

Definition 6.1. Let $n=m+2 \kappa, \triangle_{n}(\tau)$ is a (half) trigonometric polynomial of degree $n / 2$ with all simple zeros $\alpha_{n, j}(j=1,2, \cdots, n)$ lying in $[0,2 \pi), \nabla_{m}(\tau)$ is a $($ half $)$ trigonometric polynomial of degree $m / 2$ with all simple zeros $\beta_{m, j}(j=$ $1,2, \cdots, m)$ lying in $[0,2 \pi)$. We call $\left(\triangle_{n}, \nabla m\right)$ a pair of (half) trigonometric polynomials associated with (A, B), or simply, a pair of TPs, if and only if it satisfies the relationship

$$
\begin{gathered}
\mathbf{A} \triangle_{n}=\nabla_{m}, \quad \mathbf{B} \nabla_{m}=\triangle_{n}, \quad \triangle_{n} \perp_{1} H_{0}^{T}, \quad \nabla_{m} \perp_{2} H_{0}^{T}, \text { when } n \text { is even, } \\
\overline{\mathbf{A}} \triangle_{n}=\nabla_{m}, \quad \overline{\mathbf{B}} \nabla_{m}=\triangle_{n}, \quad \text { when } n \text { is odd. }
\end{gathered}
$$

There are many such pairs of TPs. Some examples may be found in 17, 21, 26 and shall be given in the final section of the present paper. It must be pointed that the conditions given in (6.1) are not independent each other. To show this, we give an example as follows.

Example 6.1. While $n=2 \ell$ and $\kappa>0$ or $\kappa=0$ with $\theta=0$, by Remark 5.4, Remark 5.5 and Remark 5.7, we know $\mathbf{B} \nabla_{m}=\triangle_{n} \Longrightarrow \mathbf{A} \triangle_{n}=\nabla_{m}, \triangle_{n} \perp_{1} H_{\kappa}^{T}\left([\theta]_{\pi}\right)$. Moreover, by Theorem 5.1 we get

$$
\begin{aligned}
\int_{0}^{2 \pi} w_{1}(\tau) \triangle_{n}(\tau) T_{r}(\tau) \mathrm{d} \tau & =\int_{0}^{2 \pi} w_{2}(\tau) \nabla_{m}(\tau)\left(\mathbf{A}^{*} T_{r}\right)(\tau) \mathrm{d} \tau \\
& =\int_{0}^{2 \pi} w_{2}(\tau) \nabla_{m}(\tau) \operatorname{Q.P}\left[X T_{r}\right](\tau) \mathrm{d} \tau .
\end{aligned}
$$

In this identity taking $T_{r} \in H_{\kappa}^{T}\left([\pi / 2+\theta]_{\pi}\right)$, by (4.8) we get $\nabla m \perp_{2} H_{0}^{T} \Longrightarrow$ $\triangle_{n} \perp_{1} H_{\kappa}^{T}\left([\pi / 2+\theta]_{\pi}\right)$, and finally $\triangle_{n} \perp_{1} H_{\kappa}^{T}$. Similarly, while $n=2 \ell$ and $\kappa<0$ or $\kappa=0$ with $\theta=0$ we get $\mathbf{B} \nabla_{m}=\triangle_{n}$ and $\nabla m \perp_{2} H_{-\kappa}^{T}$ from $\mathbf{A} \triangle_{n}=\nabla m$ and $\triangle_{n} \perp_{1} H_{0}^{T}$. While $n=2 \ell$ and $\kappa=0$ with $\theta \neq 0$, we know that, by using Theorem 5.2, we get $\triangle_{n} \perp_{1} H_{0}^{T}$ and $\nabla_{m} \perp_{2} H_{0}^{T}$ from $\mathbf{B} \nabla_{m}=\triangle_{n}$ and $\mathbf{A} \triangle_{n}=\nabla_{m}$.

Lemma 6.1. Let $n=2 \ell$ be even. If $\left(\triangle_{n}, \nabla m\right)$ is a pair of TPs, then

$$
\triangle_{n} \perp_{1} H_{\max \{0, \kappa\}}^{T}, \quad \nabla m \perp_{2} H_{\max \{0,-\kappa\}}^{T} .
$$

Proof. This follows from Example 6.1 above. 
If $h_{n}(\tau)=c \prod_{j=1}^{n} \sin \frac{\tau-t_{j}}{2}$, a half-trigonometric polynomial, where $c \neq 0$ is a constant and $t_{j}$ 's are pairwisely different points in $[0,2 \pi)$, we denote the discretization operator at the set of its zeros $t_{j}(j=1,2, \cdots, n)$ by

$$
\mathbf{r}_{n}^{h} f=\left(f\left(t_{1}\right), \cdots, f\left(t_{n}\right)\right)^{T},
$$

and the trigonometric interpolation polynomial of normal form for $f$ at the zeros $t_{j}(j=1,2, \cdots, n)$ by $\left.[26]-28\right]$,

$$
\left(L_{n}^{h} f\right)(\tau)= \begin{cases}\sum_{j=1}^{n} \frac{h_{n}(\tau)}{2 h_{n}^{\prime}\left(t_{j}\right)} \csc \frac{\tau-t_{j}}{2} f\left(t_{j}\right) \quad \text { if } n=2 \ell-1 \text { is odd }, \\ \sum_{j=1}^{n} \frac{h_{n}(\tau)}{2 h_{n}^{\prime}\left(t_{j}\right)} \cot \frac{\tau-t_{j}}{2} f\left(t_{j}\right) \quad \text { if } n=2 \ell \quad \text { is even. }\end{cases}
$$

Let

$$
\phi=\frac{\pi}{2}-\frac{1}{2} \sum_{j=1}^{n} t_{j}
$$

Obviously, by Lemma 4.1,

$$
h_{n} \in \begin{cases}H_{\ell}^{T}\left([\phi]_{\pi}\right), & \text { if } n=2 \ell \\ H_{\ell-\frac{1}{2}}^{T}\left([\pi / 2+\phi]_{\pi}\right) & \text { if } n=2 \ell-1 .\end{cases}
$$

Thus we have [27],

$$
\operatorname{ker}\left(\mathbf{I}-L_{n}^{h}\right)= \begin{cases}H_{\ell}^{T}\left([\pi / 2+\phi]_{\pi}\right), & \text { if } n=2 \ell \\ H_{\ell-1}^{T}, & \text { if } n=2 \ell-1 .\end{cases}
$$

If $\left(\triangle_{n}, \nabla_{m}\right)$ is a pair of TPs, now we construct some quadrature formulae. For the proper integral

$$
\mathbf{U} f=\frac{1}{2 \pi} \int_{0}^{2 \pi} w_{1}(\tau) f(\tau) d \tau
$$

let

$$
\mathbf{Q}_{n}^{\triangle U} f \stackrel{\text { def }}{=} \mathbf{U} L_{n}^{\triangle} f=E_{n}^{\triangle U} \mathbf{r}_{n}^{\triangle} f
$$

where

$$
\begin{gathered}
E_{n}^{\triangle U}=\left(u_{n, 1}, \cdots, u_{n, n}\right) \text { with } u_{n, j}=\frac{\triangle_{n}^{*}\left(\alpha_{n, j}\right)}{4 \pi \triangle_{n}^{\prime}\left(\alpha_{n, j}\right)}, \\
\triangle_{n}^{*}(z)=\left\{\begin{array}{l}
\int_{0}^{2 \pi} w_{1}(\tau) \triangle_{n}(\tau) \cot \frac{\tau-z}{2} d \tau, \quad \text { if } n \text { is even, } \\
\int_{0}^{2 \pi} w_{1}(\tau) \triangle_{n}(\tau) \csc \frac{\tau-z}{2} d \tau, \quad \text { if } n \text { is odd, }
\end{array}\right.
\end{gathered}
$$

$\triangle_{n}^{*}$ is called the associated function of $\triangle_{n}$ with respect to the weight $w_{1}$. We approximate $\mathbf{Q}_{n}^{\triangle U} f$ to $\mathbf{U} f$ and denote the remainder by

$$
\mathbf{R}_{n}^{\triangle U} f=\mathbf{U} f-\mathbf{Q}_{n}^{\triangle U} f .
$$

Obviously, by (6.8)

$$
\operatorname{ker}\left(\mathbf{R}_{n}^{\triangle U}\right) \supseteq\left\{\begin{array}{ll}
H_{\ell}^{T}\left(\left[\pi / 2+\phi_{1}\right]_{\pi}\right), & \text { if } n=2 \ell, \\
H_{\ell-1}^{T}, & \text { if } n=2 \ell-1,
\end{array} \quad \phi_{1}=\frac{\pi}{2}-\frac{1}{2} \sum_{j=1}^{n} \alpha_{n, j} .\right.
$$


Similarly, for the proper integral

$$
\mathbf{V} f=\frac{1}{2 \pi} \int_{0}^{2 \pi} w_{2}(\tau) f(\tau) \mathrm{d} \tau
$$

let

$$
\mathbf{Q}_{m}^{\nabla V} f \stackrel{\text { def }}{=} \mathbf{V} L_{m}^{\nabla} f=E_{m}^{\nabla V} \mathbf{r}_{m}^{\nabla} f
$$

where

$$
E_{m}^{\nabla V}=\left(v_{m, 1}, \cdots, v_{m, m}\right) \quad \text { with } \quad v_{m, j}=\frac{\nabla_{m}^{*}\left(\beta_{m, j}\right)}{4 \pi \nabla_{m}^{\prime}\left(\beta_{m, j}\right)},
$$

$\nabla_{m}^{*}$ so-called the associated function of $\nabla m$ with respect to the weight $w_{2}$ is given as follows

$$
\nabla_{m}^{*}(z)=\left\{\begin{array}{ll}
\int_{0}^{2 \pi} w_{2}(\tau) \nabla_{m}(\tau) \cot \frac{\tau-z}{2} d \tau, & \text { if } m \text { is even, } \\
\int_{0}^{2 \pi} w_{2}(\tau) \nabla_{m}(\tau) \csc \frac{\tau-z}{2} d \tau, & \text { if } m \text { is odd, }
\end{array} \quad z \in \mathcal{C} .\right.
$$

Definition 6.2. If $H_{k-1}^{T} \subseteq \operatorname{ker}\left(\mathbf{R}_{n}^{\triangle U}\right)$, but there exists some $T_{k} \in H_{k}^{T}$ such that $\mathbf{R}_{n}^{\triangle U} T_{k} \neq 0$, then we define the quadrature formula (6.13) to have trigonometric precision of order $(k-1)$, denoted as $\mathbf{p r}\left(\mathbf{Q}_{n}^{\triangle U}\right)=k-1$.

Definition 6.3. If $\operatorname{pr}\left(\mathbf{Q}_{n}^{\triangle U}\right)=k-1$ and $H_{k}^{T}(\alpha) \subseteq \operatorname{ker}\left(\mathbf{R}_{n}^{\triangle U}\right)$, we say (6.13) is of $H_{k}^{T}(\alpha)$ type, denoted as $\mathbf{t y}\left(\mathbf{Q}_{n}^{\triangle U}\right)=H_{k}^{T}(\alpha)$.

The two definitions above are seen in [27] in detail. The meanings of the $\operatorname{pr}\left(\mathbf{Q}_{n}^{\nabla V}\right)$ and $\mathbf{t y}\left(\mathbf{Q}_{m}^{\nabla V}\right)$ are similar and obvious. Now we know the following result from (6.8).

Lemma 6.2. $\operatorname{pr}\left(\mathbf{Q}_{n}^{\triangle U}\right) \geq\left[\frac{n-1}{2}\right]$ and $\operatorname{pr}\left(\mathbf{Q}_{m}^{\nabla V}\right) \geq\left[\frac{m-1}{2}\right]$ where $[x]$ is the integer part of $x$. When $n=2 \ell$ is even, then $\mathbf{t y}\left(\mathbf{Q}_{n}^{\triangle U}\right) \supseteq H_{\ell}^{T}\left(\left[\pi / 2+\phi_{1}\right]_{\pi}\right)$ and $\mathbf{t y}\left(\mathbf{Q}_{m}^{\nabla V}\right) \supseteq$ $H_{\ell-\kappa}^{T}\left(\left[\pi / 2+\phi_{2}\right]_{\pi}\right)$ where $\phi_{1}$ is given in (6.14) and

$$
\phi_{2}=\frac{\pi}{2}-\frac{1}{2} \sum_{j=1}^{m} \beta_{m, j} .
$$

Since $\left(\triangle_{n}, \nabla_{m}\right)$ is a pair of TPs, we have a better result.

\section{Lemma 6.3.}

$$
\operatorname{pr}\left(\mathbf{Q}_{n}^{\triangle U}\right) \geq \max \left\{\left[\frac{n}{2}\right],\left[\frac{n}{2}\right]+\kappa\right\} \text { and } \mathbf{p r}\left(\mathbf{Q}_{m}^{\nabla V}\right) \geq \max \left\{\left[\frac{m}{2}\right],\left[\frac{m}{2}\right]-\kappa\right\} .
$$

Proof. We only prove the first conclusion. When $n=2 \ell-1$ and $\kappa>0$, noting that $\triangle_{n} \in H_{\ell-\frac{1}{2}}^{T *}$ and using the Euclidean division formula in Lemma 4.3, we get $H_{\ell+\kappa-1}^{T}=\triangle_{n} H_{\kappa-\frac{1}{2}}^{T} \oplus H_{\ell-1}^{T}$. So, by Lemma 6.2 and (5.13) we know $\mathbf{R}_{m}^{\triangle U}\left(H_{\ell+\kappa-1}^{T}\right)=$ $\{0\}$. When $n=2 \ell$, noting that $\triangle_{n} \in H_{\ell}^{T *}\left(\left[\phi_{1}\right]_{\pi}\right)$ and using the Euclidean division formula, we get $H_{\ell+\max \{0, \kappa\}}^{T}=\triangle_{n} H_{\max \{0, \kappa\}}^{T} \oplus H_{\ell}^{T}\left(\left[\pi / 2+\phi_{1}\right]_{\pi}\right)$. Thus, by Lemma 6.1 and Lemma 6.2 we know $\mathbf{R}_{n}^{\triangle U}\left(H_{\ell+\max \{0, \kappa\}}^{T}\right)=\{0\}$. 
Now we set up the quadrature formulae of $\mathbf{D}_{1}$ and $\mathbf{D}_{2}$, respectively,

$$
\begin{aligned}
& \left(\mathbf{Q}_{n}^{\triangle D_{1}} f\right)(t)=E_{n}^{\triangle D_{1}}(t) \mathbf{r}_{n}^{\triangle} f \\
& \quad \equiv\left(u_{n, 1} d_{1}\left(\alpha_{n, 1}, t\right), u_{n, 2} d_{1}\left(\alpha_{n, 2}, t\right), \cdots, u_{n, n} d_{1}\left(\alpha_{n, n}, t\right)\right) \mathbf{r}_{n}^{\triangle} f, \\
& \left(\mathbf{Q}_{m}^{\nabla D_{2}} f\right)(t)=E_{m}^{\nabla D_{2}}(t) \mathbf{r}_{m}^{\nabla} f \\
& \quad \equiv\left(v_{m, 1} d_{2}\left(\beta_{m, 1}, t\right), v_{m, 2} d_{2}\left(\beta_{m, 2}, t\right), \cdots, v_{m, m} d_{2}\left(\beta_{n, n}, t\right)\right) \mathbf{r}_{m}^{\nabla} f .
\end{aligned}
$$

Lemma 6.4. $\operatorname{pr}\left(\mathbf{Q}_{n}^{\triangle D_{1}}\right) \geq\left[\frac{n}{2}\right]$ and $\operatorname{pr}\left(\mathbf{Q}_{m}^{\nabla D_{2}}\right) \geq\left[\frac{m}{2}\right]$, where the definitions $\operatorname{pr}\left(\mathbf{Q}_{n}^{\triangle D_{1}}\right)$ and $\mathbf{p r}\left(\mathbf{Q}_{m}^{\nabla D_{2}}\right)$ are similar to Definition 6.2. In particular, $\mathbf{Q}_{n}^{\triangle D_{1}} f=$ $\mathbf{D}_{1} L_{n}^{\triangle} f, \mathbf{Q}_{m}^{\triangle D_{2}} f=\mathbf{D}_{2} L_{m}^{\nabla} f$.

Proof. When $\kappa<0$ and $\kappa=0$ with $[\theta]_{\pi}=0$, since $\mathbf{D}_{1}=0$ and $\mathbf{Q}_{n}^{\triangle D_{1}}=0$ by (5.6) and Remark 5.4, we agree that $\operatorname{pr}\left(\mathbf{Q}_{n}^{\triangle D_{1}}\right)=+\infty$, thus the conclusion required is trivial. For the case of $\kappa>0$ and $\kappa=0$ with $[\theta]_{\pi} \neq 0$, by Lemma 6.3 and (5.5), the conclusions required follow.

From [27] we may obtain the singular quadrature operators (SQOs) $\mathbf{Q}_{n}^{\triangle A}$ for $\mathbf{A}$ and $\mathbf{Q}_{m}^{\nabla B}$ for $\mathbf{B}$.

$$
\left(\mathbf{Q}_{n}^{\triangle A} f\right)(t)=\frac{\nabla_{m}(t)}{\triangle_{n}(t)} f(t)+b(t) E_{n}^{\triangle A}(t) \mathbf{r}_{n}^{\triangle} f
$$

where

$$
E_{n}^{\triangle A}(t)=\left(u_{n, 1} \cot \frac{\alpha_{n, 1}-t}{2}, u_{n, 2} \cot \frac{\alpha_{n, 2}-t}{2}, \cdots, u_{n, n} \cot \frac{\alpha_{n, n}-t}{2}\right),
$$

and

$$
\left(\mathbf{Q}_{m}^{\nabla B} f\right)(t)=\frac{\triangle_{n}(t)}{\nabla_{m}(t)} f(t)-b(t) E_{m}^{\nabla B}(t) \mathbf{r}_{m}^{\nabla} f
$$

where

$$
E_{m}^{\nabla B}(t)=\left(v_{m, 1} \cot \frac{\beta_{m, 1}-t}{2}, v_{m, 2} \cot \frac{\beta_{m, 2}-t}{2}, \cdots, v_{m, m} \cot \frac{\beta_{m, m}-t}{2}\right) .
$$

Definition 6.4. While $\left(\triangle_{n}, \nabla_{m}\right)$ is a pair of TPs of SIOs $(\mathbf{A}, \mathbf{B})$, then $\left(\mathbf{Q}_{n}^{\triangle A}, \mathbf{Q}_{m}^{\nabla B}\right)$ given by (6.22) and (6.24) is said to be a pair of associated SQOs relative to $(\mathbf{A}, \mathbf{B})$.

Remark 6.1. We easily obtain

$$
\begin{gathered}
u_{n, j} b\left(\alpha_{n, j}\right)=\frac{\nabla_{m}\left(\alpha_{n, j}\right)}{2 \triangle_{n}^{\prime}\left(\alpha_{n, j}\right)}(j=1,2, \cdots, n), \\
v_{m, k} b\left(\beta_{m, k}\right)=\frac{\triangle_{n}\left(\beta_{m, k}\right)}{2 \nabla_{m}^{\prime}\left(\beta_{m, k}\right)}(k=1,2, \cdots, m) .
\end{gathered}
$$

For example, by (6.1) and (6.11) we get

$$
\nabla m\left(\alpha_{n, j}\right)=\frac{1}{2 \pi} b\left(\alpha_{n, j}\right) \triangle_{n}^{*}\left(\alpha_{n, j}\right)=2 \triangle_{n}^{\prime}\left(\alpha_{n, j}\right) b\left(\alpha_{n, j}\right) u_{n, j}
$$

Let $C_{2 \pi}^{\prime}\left(h_{n}\right)$ denote the family of continuous functions with period $2 \pi$ and possessing derivates at the zeros of $h_{n}$. By Remark 6.1 we may obtain the following conclusion.

Remark 6.2. $\mathbf{Q}_{n}^{\triangle A}: C_{2 \pi}^{\prime}\left(\triangle_{n}\right) \rightarrow C_{2 \pi}^{\prime}(\nabla m)$ and $\mathbf{Q}_{m}^{\nabla B}: C_{2 \pi}^{\prime}(\nabla m) \rightarrow C_{2 \pi}^{\prime}\left(\triangle_{n}\right)$. 
From [27] or directly using Lemma 6.3, we also obtain the following result.

Lemma 6.5. $\operatorname{pr}\left(\mathbf{Q}_{n}^{\triangle A}\right) \geq\left[\frac{n}{2}\right]+\max \{0, \kappa\}$ and $\operatorname{pr}\left(\mathbf{Q}_{m}^{\nabla B}\right) \geq\left[\frac{m}{2}\right]+\max \{0,-\kappa\}$.

Now we assume that $n$ is sufficiently large. The following theorem is completely parallel to Theorem 5.2.

Theorem 6.1. $\quad \mathbf{Q}_{n}^{\triangle A} \mathbf{Q}_{m}^{\nabla B}=\mathbf{I}+b \mathbf{Q}_{m}^{\nabla D_{2}}, \quad \mathbf{Q}_{m}^{\nabla B} \mathbf{Q}_{n}^{\triangle A}=\mathbf{I}-b \mathbf{Q}_{n}^{\triangle D_{1}}$.

Proof. Only prove the second equality. The compositions of $\mathbf{Q}_{n}^{\triangle A}$ and $\mathbf{Q}_{m}^{\nabla B}$ are just reasonable by Remark 6.2. First suppose $f \in \operatorname{ker}\left(\mathbf{r}_{n}^{\triangle}\right)$, we get $\mathbf{Q}_{m}^{\nabla} \mathbf{Q}_{n}^{\triangle A} f=f$. For a general function $f$, we then have

$$
\begin{aligned}
\mathbf{Q}_{m}^{\nabla B} \mathbf{Q}_{n}^{\triangle A} f & =f-L_{n}^{\triangle} f+\mathbf{B A} L_{n}^{\triangle} f \quad \text { (by Lemma } 6.5 \text { and Theorem 5.4) } \\
& =f-L_{n}^{\triangle} f+\left(\mathbf{I}-b \mathbf{D}_{1}\right) L_{n}^{\triangle} f \quad \text { (by Theorem 5.2) } \\
& =f-b \mathbf{Q}_{n}^{\triangle D_{1}} f \quad(\text { by Lemma } 6.4) .
\end{aligned}
$$

Remark 6.3. If $\kappa>0$, then $\mathbf{Q}_{m}^{\nabla D_{2}}=0$ by (5.5), this is to say that $\mathbf{Q}_{n}^{\triangle A}$ has the right inverse $\mathbf{Q}_{m}^{\nabla B}$. If $\kappa<0$, then $\mathbf{Q}_{n}^{\triangle D_{1}}=0$ by (5.5), this is to say that $\mathbf{Q}_{n}^{\triangle A}$ has the left inverse $\mathbf{Q}_{m}^{\nabla B}$. If $\kappa=0$, then $\mathbf{Q}_{n}^{\triangle D_{1}}=-\sin \theta \mathbf{Q}_{n}^{\triangle U}$ and $\mathbf{Q}_{m}^{\nabla D_{2}}=\sin \theta \mathbf{Q}_{m}^{\nabla V}$ by $(5.6)$.

Using Theorem 6.1 and Lemma 6.5, we may get the following results, which are parallel to those in the last section and their proofs follow trivially in a completely analogous way to that used there.

Corollary 6.1. $\operatorname{Ima}\left(\mathbf{Q}_{n}^{\triangle D_{1}}\right)=H_{\kappa}^{T}\left([\theta]_{\pi}\right)$ and $\operatorname{Ima}\left(\mathbf{Q}_{m}^{\nabla D_{2}}\right)=H_{-\kappa}^{T}\left([-\theta]_{\pi}\right)$. If $\kappa \neq 0$ or $\kappa=0$ with $[\theta]_{\pi}=\pi / 2$, then $\operatorname{ker}\left(\mathbf{Q}_{n}^{\triangle A}\right)=b H_{\kappa}^{T}\left([\theta]_{\pi}\right), \operatorname{ker}\left(\mathbf{Q}_{m}^{\nabla}\right)=b H_{-\kappa}^{T}\left([-\theta]_{\pi}\right)$, $\mathbf{Q}_{n}^{\triangle D_{1}} b T_{\kappa}=T_{\kappa}$ when $T_{\kappa} \in H_{\kappa}^{T}\left([\theta]_{\pi}\right), \mathbf{Q}_{m}^{\nabla D_{2}} b T_{-\kappa}=-T_{-\kappa}$ when $T_{-\kappa} \in H_{-\kappa}^{T}\left([-\theta]_{\pi}\right)$. If $\kappa=0$ with $[\theta]_{\pi} \neq \pi / 2$, then $\operatorname{ker}\left(\mathbf{Q}_{n}^{\triangle A}\right)=\operatorname{ker}\left(\mathbf{Q}_{m}^{\nabla B}\right)=\{0\}$.

Corollary 6.2. If $\kappa \neq 0$ or $\kappa=0$ with $[\theta]_{\pi}=\pi / 2$, then $b \mathbf{Q}_{n}^{\triangle D_{1}}$ and $-b \mathbf{Q}_{m}^{\nabla D_{2}}$ are idempotent, $\mathbf{Q}_{n}^{\triangle D_{1}}$ and $\mathbf{Q}_{m}^{\nabla D_{2}}$ are right zero divisors of $\mathbf{Q}_{m}^{\nabla B}$ and $\mathbf{Q}_{n}^{\triangle A}$, respectively, $b \mathbf{Q}_{n}^{\triangle D_{1}}$ and $b \mathbf{Q}_{m}^{\nabla D_{2}}$ are left zero divisors of $\mathbf{Q}_{n}^{\triangle A}$ and $\mathbf{Q}_{m}^{\nabla}$, respectively, i.e., $\mathbf{Q}_{n}^{\triangle D_{1}} \mathbf{Q}_{m}^{\nabla B}=0, \mathbf{Q}_{m}^{\nabla D_{2}} \mathbf{Q}_{n}^{\triangle A}=0, \mathbf{Q}_{n}^{\triangle A} b \mathbf{Q}_{n}^{\triangle D_{1}}=0$ and $\mathbf{Q}_{m}^{\nabla} b \mathbf{Q}_{m}^{\nabla D_{2}}=0$.

Theorem 6.2. If $\kappa>0$ and $N_{\kappa} \in H_{\kappa}^{T}\left([\theta]_{\pi}\right)$ is a given trigonometric polynomial, then under the condition $\mathbf{Q}_{n}^{\triangle D_{1}} y=N_{\kappa}, \mathbf{Q}_{n}^{\triangle A} y=f$ possesses the unique solution $y=\mathbf{Q}_{m}^{\nabla B} f+b N_{\kappa}$. If $\kappa<0$, the condition of solvability for $\mathbf{Q}_{n}^{\triangle A} y=f$ is $\mathbf{Q}_{m}^{\nabla D_{2}} f=0$ and it possesses the unique solution $y=\mathbf{Q}_{m}^{\nabla} f$ when it is fulfilled. If $\kappa=0$ with $[\theta]_{\pi}=\pi / 2$, the condition of solvability for $\mathbf{Q}_{n}^{\triangle A} y=f$ is $\mathbf{Q}_{m}^{\nabla D_{2}} f=0$ and when it is fulfilled it possesses the unique solution $y=\mathbf{Q}_{m}^{\nabla} f+b N$ under the condition $\mathbf{Q}_{n}^{\triangle D_{1}} y=N$ where $N$ is a given constant. If $\kappa=0$ with $[\theta]_{\pi} \neq \pi / 2$, then $\mathbf{Q}_{n}^{\triangle A} y=f$ possesses the unique solution $y=\mathbf{Q}_{m}^{\nabla} f-b \sec \theta \mathbf{Q}_{m}^{\nabla D_{2}} f$.

Remark 6.4. If $\kappa=0$ with $\theta \neq \pi / 2$, then we know $\left(\mathbf{Q}_{n}^{\triangle A}\right)^{-1}=\mathbf{Q}_{m}^{\nabla B}-b \sec \theta \mathbf{Q}_{m}^{\nabla D_{2}}=$ $\mathbf{Q}_{m}^{\nabla} B-b \tan \theta \mathbf{Q}_{m}^{\nabla V}$, by Corollary 6.1 and Theorem 6.2.

\section{Discretization matrices}

In this section, we discuss the compositions of the associated SQOs and the discretization operators. Applying $\mathbf{r}_{m}^{\nabla}$ to $\mathbf{Q}_{n}^{\triangle A}$ we get

$$
\mathbf{r}_{m}^{\nabla} \mathbf{Q}_{n}^{\triangle A} f=A_{m, n} \mathbf{r}_{n}^{\triangle} f
$$


where the $(m, n)$ matrix $A_{m, n}$ is just

$$
A_{m, n}=\left(a_{k, j}\right), \quad a_{k, j}= \begin{cases}b\left(\beta_{m, k}\right) u_{n, j} \cot \frac{\alpha_{n, j}-\beta_{m, k}}{2}, & \text { if } \alpha_{n, j} \neq \beta_{m, k}, \\ \frac{\nabla_{m}^{\prime}\left(\beta_{m, k}\right)}{\triangle_{n}^{\prime}\left(\alpha_{n, j}\right)}-2 b^{\prime}\left(\beta_{m, k}\right) u_{n, j}, & \text { if } \alpha_{n, j}=\beta_{m, k} .\end{cases}
$$

In (7.2), the case $\alpha_{n, j} \neq \beta_{m, k}$ is obvious. If $\alpha_{n, j}=\beta_{m, k}$, from Remark 6.1 we know $b\left(\beta_{m, k}\right) u_{n, j}=0$, so,

$$
\begin{aligned}
a_{k, j} & =\lim _{t \rightarrow \beta_{m, k}}\left[\frac{\nabla_{m}(t)}{\triangle_{n}(t)}+b(t) u_{n, j} \cot \frac{\alpha_{n, j}-t}{2}\right] \\
& =\frac{\nabla_{m}^{\prime}\left(\beta_{m, k}\right)}{\triangle_{n}^{\prime}\left(\alpha_{n, j}\right)}-2 b^{\prime}\left(\beta_{m, k}\right) u_{n, j} .
\end{aligned}
$$

Remark 7.1. If $\alpha_{n, j}=\beta_{m, k}$ and $u_{n, j} \neq 0$, then $a_{k, j}=a\left(\alpha_{n, j}\right) w_{1}\left(\alpha_{n, j}\right)$, from (6.1) and (7.3).

The matrix $A_{m, n}$ arises from discretizing $\mathbf{Q}_{n}^{\triangle A}$ by $\mathbf{r}_{m}^{\nabla}$. We call it the discretization matrix of $\mathbf{Q}_{n}^{\triangle A}$. By analogy, discretizing $\mathbf{Q}_{m}^{\nabla B}$ by $\mathbf{r}_{n}^{\triangle}$, we also have

$$
\mathbf{r}_{n}^{\triangle} \mathbf{Q}_{m}^{\nabla B} f=B_{n, m} \mathbf{r}_{m}^{\nabla} f
$$

where the $(n, m)$ matrix $B_{n, m}$ called the discretization matrix of $\mathbf{Q}_{m}^{\nabla B}$ is

$$
B_{n, m}=\left(b_{j, r}\right), \quad b_{j, r}= \begin{cases}b\left(\alpha_{n, j}\right) v_{m, r} \cot \frac{\alpha_{n, j}-\beta_{m, r}}{2}, & \text { if } \alpha_{n, j} \neq \beta_{m, r}, \\ \frac{\triangle_{n}^{\prime}\left(\alpha_{n, j}\right)}{\nabla_{m}^{\prime}\left(\beta_{m, r}\right)}+2 b^{\prime}\left(\alpha_{n, j}\right) v_{m, r}, & \text { if } \alpha_{n, j}=\beta_{m, r} .\end{cases}
$$

Remark 7.2. If $\alpha_{n, j}=\beta_{m, r}$ and $v_{m, r} \neq 0$, then $b_{j, r}=a\left(\beta_{m, r}\right) w_{2}\left(\beta_{m, r}\right)$.

Definition 7.1. $\left(A_{m, n}, B_{n, m}\right)$ in (7.2) and (7.5) is called the pair of associated matrices of $\left(\mathbf{Q}_{n}^{\triangle A}, \mathbf{Q}_{m}^{\nabla B}\right)$.

Lemma 7.1. Let $\left(A_{m, n}, B_{n, m}\right)$ be the pair of associated matrices and let $I_{k}$ denote the unit square matrix of order $k$. Then $A_{m, n} B_{n, m}=I_{m}$ when $\kappa>0$ and $B_{n, m} A_{m, n}=I_{n}$ when $\kappa<0$.

Proof. We only prove the first equality. From Remark 6.3, we have $\mathbf{Q}_{n}^{\triangle A} \mathbf{Q}_{m}^{\nabla B} f=$ $f$. Applying $\mathbf{r}_{m}^{q}$ to both sides of this equality, we get $\mathbf{r}_{m}^{\nabla} f=\mathbf{r}_{m}^{\nabla} \mathbf{Q}_{n}^{\triangle A} \mathbf{Q}_{m}^{\nabla B} f=$ $A_{m, n} \mathbf{r}_{n}^{\triangle} \mathbf{Q}_{m}^{\nabla B} f=A_{m, n} B_{n, m} \mathbf{r}_{m}^{\nabla} f$ by (7.1) and (7.4). Noting that $\mathbf{r}_{m}^{\nabla} f$ is arbitrary, finally, $A_{m, n} B_{n, m}=I_{m}$.

First, we treat the case of $\kappa>0$. We arbitrarily choose $2 \kappa$ different points $\beta_{m, m+k}(k=1,2, \cdots, 2 \kappa)$ in $[0,2 \pi)$, only requiring that

$$
\vartheta_{2}=\left[\frac{\pi}{2}-\frac{1}{2} \sum_{k=1}^{2 \kappa} \beta_{m, m+k}\right]_{\pi} \neq[\theta]_{\pi} .
$$

Let

$$
\vee_{2 \kappa}(\tau)=\prod_{k=1}^{2 \kappa} \sin \frac{\tau-\beta_{m, m+k}}{2}
$$


Then the trigonometric interpolation of $f$ in $H_{\kappa}^{T}\left([\theta]_{\pi}\right)$ on the zeros of $\vee_{2 \kappa}$ is (e.g. see (2.4) in [27])

$$
\begin{aligned}
& \left(L_{2 \kappa}^{\vee} f\right)(\tau)=\sum_{k=1}^{2 \kappa} \vee_{\kappa, k}(\tau) f\left(\beta_{m, m+k}\right) \\
& \quad \text { with } \vee_{\kappa, k}(\tau)=\frac{\vee_{2 \kappa}(\tau) \sin \left(\frac{\tau-\beta_{m, m+k}}{2}+\theta-\vartheta_{2}\right)}{\vee_{2 \kappa}^{\prime}\left(\beta_{m, m+k}\right) \sin \left(\theta-\vartheta_{2}\right)} \csc \frac{\tau-\beta_{m, m+k}}{2} .
\end{aligned}
$$

Discretizing $\mathbf{Q}_{n}^{\triangle D_{1}}$ by the discretization operator $\mathbf{r}_{2 \kappa}^{\vee}$ at the set of zeros of $\vee_{2 \kappa}$, we get

$$
\mathbf{r}_{2 \kappa}^{\vee} \mathbf{Q}_{n}^{\triangle D_{1}} f=A_{2 \kappa, n} \mathbf{r}_{n}^{\triangle} f
$$

where the $(2 \kappa, n)$ matrix $A_{2 \kappa, n}$ is

$$
A_{2 \kappa, n}=\left(a_{m+k, j}\right) \quad \text { with } \quad a_{m+k, j}=u_{n, j} d_{1}\left(\alpha_{n, j}, \beta_{m, m+k}\right) .
$$

Applying $\mathbf{r}_{n}^{\triangle}$ to $b L_{2 \kappa}^{\vee} f$ we get

$$
\mathbf{r}_{n}^{\triangle}\left(b L_{2 \kappa}^{\vee} f\right)=B_{n, 2 \kappa} \mathbf{r}_{2 \kappa}^{\vee} f
$$

where the $(n, 2 \kappa)$ matrix $B_{n, 2 \kappa}$ is

$$
B_{n, 2 \kappa}=\left(b_{j, m+r}\right) \text { with } b_{j, m+r}=b\left(\alpha_{n, j}\right) \vee_{\kappa, r}\left(\alpha_{n, j}\right) .
$$

Let the partitioned matrices (square matrices of order $n$ )

$$
A_{n}=\left(\begin{array}{c}
A_{m, n} \\
A_{2 \kappa, n}
\end{array}\right), \quad B_{n}=\left(B_{n, m}, B_{n, 2 \kappa}\right) .
$$

Lemma 7.2. Let $\kappa>0, A_{n}$ and $B_{n}$ be as above, then $A_{n} B_{n}=I_{n}$.

So $A_{n}$ and $B_{n}$ are called the supplemented matrices of $A_{m, n}$ and $B_{n, m}$, respectively.

Proof. By Corollary 6.1 and noting $L_{2 \kappa}^{\vee} f \in H_{\kappa}^{T}\left([\theta]_{\pi}\right)$, we have $\mathbf{Q}_{n}^{\triangle D_{1}}\left(b L_{2 \kappa}^{\vee} f\right)=$ $L_{2 \kappa}^{\vee} f$. Thus, by (7.9) and (7.11) we get $\mathbf{r}_{2 \kappa}^{\vee} f=\mathbf{r}_{2 \kappa}^{\vee}\left(L_{2 \kappa}^{\vee} f\right)=\mathbf{r}_{2 \kappa}^{\vee} \mathbf{Q}_{n}^{\triangle D_{1}}\left(b L_{2 \kappa}^{\vee} f\right)=$ $A_{2 \kappa, n} \mathbf{r}_{n}^{\triangle}\left(b L_{2 \kappa}^{\vee} f\right)=A_{2 \kappa, n} B_{n, 2 \kappa} \mathbf{r}_{2 \kappa}^{\vee} f$. Noting that $\mathbf{r}_{2 \kappa}^{\vee} f$ is arbitrary, finally, we get $A_{2 \kappa, n} B_{n, 2 \kappa}=I_{2 \kappa}$. Let $O_{k, j}$ denote the $(k, j)$ zero matrix. By Corollary 6.1, (7.1) and (7.11), we have $O_{n, 1}=\mathbf{r}_{m}^{\nabla} \mathbf{Q}_{n}^{\triangle A}\left(b L_{2 \kappa}^{\vee} f\right)=A_{n, m} \mathbf{r}_{n}^{\triangle}\left(b L_{2 \kappa}^{\vee} f\right)=A_{n, m} B_{m, 2 \kappa} \mathbf{r}_{2 \kappa}^{\vee} f$, i.e., $A_{n, m} B_{m, 2 \kappa}=O_{n, 2 \kappa}$. By Corollary 6.2, (7.9) and (7.4) we get $O_{2 \kappa, 1}$ $=\mathbf{r}_{2 \kappa}^{\vee} \mathbf{Q}_{n}^{\triangle D_{1}} \mathbf{Q}_{m}^{\nabla B} f=A_{2 \kappa, n} \mathbf{r}_{n}^{\triangleleft} \mathbf{Q}_{m}^{\nabla B} f=A_{2 \kappa, n} B_{n, m} \mathbf{r}_{m}^{\nabla} f$, so $A_{2 \kappa, n} B_{n, m}=O_{2 \kappa, m}$. Finally, noting Lemma 7.1, the proof is completed.

By analogy, when $\kappa<0$, we arbitrarily choose $(-2 \kappa)$ different points $\alpha_{n, n+k}$ $(k=1, \cdots,-2 \kappa)$ in $[0,2 \pi)$ with

$$
\vartheta_{1}=\left[\frac{\pi}{2}-\frac{1}{2} \sum_{k=1}^{-2 \kappa} \alpha_{n, n+k}\right]_{\pi} \neq[-\theta]_{\pi},
$$

and set

$$
\wedge_{-2 \kappa}(\tau)=\prod_{k=1}^{-2 \kappa} \sin \frac{\tau-\alpha_{n, n+k}}{2}
$$


We know that the trigonometric interpolation of $f$ in $H_{-\kappa}^{T}\left([-\theta]_{\pi}\right)$ on the zeros of $\wedge_{-2 \kappa}$ is 27 ]

$$
\begin{aligned}
\left(L_{-2 \kappa}^{\wedge} f\right)(\tau) & =\sum_{k=1}^{-2 \kappa} \wedge_{-\kappa, k}(\tau) f\left(\alpha_{n, n+k}\right) \\
\text { with } & \wedge_{-\kappa, k}(\tau)=\frac{\wedge_{-2 \kappa}(\tau) \sin \left(\frac{\tau-\alpha_{n, n+k}}{2}-\theta-\vartheta_{1}\right)}{\wedge_{-2 \kappa}^{\prime}\left(\alpha_{n, n+k}\right) \sin \left(-\theta-\vartheta_{1}\right)} \csc \frac{\tau-\alpha_{n, n+k}}{2} .
\end{aligned}
$$

Discretizing $\mathbf{Q}_{n}^{\triangle D_{2}}$ by the discretization operator $\mathbf{r}_{-2 \kappa}^{\wedge}$ at the set of zeros of $\wedge_{-2 \kappa}$, we get

$$
\mathbf{r}_{-2 \kappa}^{\wedge} \mathbf{Q}_{m}^{\nabla D_{2}} f=B_{-2 \kappa, m} \mathbf{r}_{m}^{\nabla} f
$$

where the $(-2 \kappa, m)$ matrix

$$
B_{-2 \kappa, m}=\left(b_{n+j, r}\right) \quad \text { with } \quad b_{n+j, r}=v_{m, r} d_{2}\left(\alpha_{n, n+j}, \beta_{m, r}\right) .
$$

From

$$
\mathbf{r}_{m}^{\nabla}\left(-b L_{-2 \kappa}^{\wedge} f\right)=A_{m,-2 \kappa} \mathbf{r}_{-2 \kappa}^{\wedge} f,
$$

we get $(m,-2 \kappa)$ matrix

$$
A_{m,-2 \kappa}=\left(a_{k, n+j}\right), \quad a_{k, n+j}=-b\left(\beta_{m, k}\right) \wedge_{-\kappa, j}\left(\beta_{m, k}\right) .
$$

Let

$$
B_{m}=\left(\begin{array}{c}
B_{n, m} \\
B_{-2 \kappa, m}
\end{array}\right), \quad A_{m}=\left(A_{m, n}, A_{m,-2 \kappa}\right) .
$$

In exactly the same way, we may prove $B_{m} A_{m}=I_{m}$. So, we still call $A_{m}$ and $B_{m}$ the supplemented matrices of $A_{m, n}$ and $B_{n, m}$, respectively.

Lemma 7.3. Let $\kappa<0, A_{m}$ and $B_{m}$ be given by (7.21), then $A_{m} B_{m}=I_{m}$.

When $\kappa=0(n=m)$ with $[\theta]_{\pi} \neq \pi / 2, A_{m, n}$ given in (7.2) is a square matrix, denoted as $A_{n}$. But we use $B_{n}$ in the present case to denote the following square matrix

$$
B_{n}=B_{n, m}-\tan \theta\left(\mathbf{r}_{n}^{\triangle} b\right) E_{m}^{\nabla V}=\left\{b_{j, r}-\tan \theta v_{m, r} b\left(\alpha_{n, j}\right)\right\},
$$

where $B_{n, m}$ and $E_{m}^{\nabla V}$ are given in (7.5) and (6.17), respectively, which is called the supplemented matrix of $B_{n, m}$. Obviously, by Remark 6.4

$$
\mathbf{r}_{n}^{\triangle}\left(\mathbf{Q}_{m}^{\nabla B}-\sec \theta b \mathbf{Q}_{m}^{\nabla D_{2}}\right) f=B_{n} \mathbf{r}_{m}^{\nabla} f .
$$

Thus, by Remark 6.4 one can show $\mathbf{r}_{n}^{\triangle} f=\mathbf{r}_{n}^{\triangle}\left(\mathbf{Q}_{m}^{\nabla B}-\sec \theta b \mathbf{Q}_{m}^{\nabla D_{2}}\right) \mathbf{Q}_{n}^{\triangle A} f=$ $B_{n} \mathbf{r}_{m}^{\nabla} \mathbf{Q}_{n}^{\triangle A} f=B_{n} A_{n} \mathbf{r}_{n}^{\triangle} f$, i.e., $B_{n} A_{n}=I_{n}$. Thus, we also have the following result.

Lemma 7.4. If $\kappa=0$ with $[\theta]_{\pi} \neq \pi / 2$, then $A_{n} B_{n}=I_{n}$ where $B_{n}$ is given in (7.22) and $A_{n}=A_{m, n}$ in (7.2).

When $\kappa=0(n=m)$ with $[\theta]_{\pi}=\pi / 2(\sin \theta= \pm 1)$, let

$$
A_{n+1, n}=\left(\begin{array}{c}
A_{m, n} \\
E_{n}^{\triangle U}
\end{array}\right), \quad B_{n, n+1}=\left(B_{n, m},-\sin \theta \mathbf{r}_{n}^{\triangle} b\right),
$$


where $A_{m, n}, E_{n}^{\triangle U}$ and $B_{n, m}$ are, respectively, given in (7.2), (6.11) and (7.5). Let

$$
A_{n+1,1}=\left(\begin{array}{c}
-\sin \theta \mathbf{r}_{m}^{\nabla b} \\
0
\end{array}\right), \quad B_{1, n+1}=\left(E_{m}^{\nabla V}, 0\right),
$$

where $E_{m}^{\nabla V}$ is still given in (6.17). Finally, we set

$$
A_{n+1}=\left(A_{n+1, n}, A_{n+1,1}\right), \quad B_{n+1}=\left(\begin{array}{c}
B_{n, n+1} \\
B_{1, n+1}
\end{array}\right),
$$

which are called the supplement matrices of $A_{m, n}$ and $B_{n, m}$, respectively.

Lemma 7.5. If $\kappa=0$ with $[\theta]_{\pi}=\pi / 2$, then $A_{n+1} B_{n+1}=I_{n+1}$ where $A_{n+1}$ and $B_{n+1}$ are given in (7.26).

Proof. (I) $\mathbf{r}_{n}^{\triangle} f=\mathbf{r}_{n}^{\triangle}\left[\mathbf{Q}_{m}^{\nabla B} \mathbf{Q}_{n}^{\triangle A}+b \mathbf{Q}_{n}^{\triangle D_{1}}\right] f=\left[B_{n, m} A_{m, n}-\sin \theta\left(\mathbf{r}_{n}^{\triangle} b\right) E_{n}^{\triangle U}\right] \mathbf{r}_{n}^{\triangle} f=$ $B_{n, n+1} A_{n+1, n} \mathbf{r}_{n}^{\triangle} f$ by Theorem 6.1, Lemma 6.3 and (7.24), i.e., $B_{n, n+1} A_{n+1, n}=I_{n}$. (II) By Corollary 6.1, (7.4), (7.24) and (7.25) we may get $O_{n, 1}=-\sin \theta \mathbf{r}_{n}^{\triangleleft} \mathbf{Q}_{m}^{\nabla B} b=$ $-\sin \theta B_{n, m} \mathbf{r}_{m}^{\nabla} b=B_{n, n+1} A_{n+1,1}$. (III) By Corollary 6.2, Remark 6.4, (7.24) and (7.25) we have $0=\mathbf{Q}_{m}^{\nabla D_{2}} \mathbf{Q}_{n}^{\triangle A} f=\sin \theta E_{m}^{\nabla V} \mathbf{r}_{m}^{\nabla} \mathbf{Q}_{n}^{\triangle A} f=\sin \theta E_{m}^{\nabla V} A_{m, n} \mathbf{r}_{n}^{\triangle} f=$ $-\sin \theta B_{1, n+1} A_{n+1, n} \mathbf{r}_{n}^{\triangle}$ f, i.e., $B_{1, n+1} A_{n+1, n}=O_{1, n}$. (IV) We have $1=-\mathbf{Q}_{m}^{\nabla D^{2}} b=$ $-\sin \theta E_{m}^{\nabla V} \mathbf{r}_{m}^{\nabla} b=B_{1, n+1} A_{n+1,1}$ by Corollary 6.1, (7.24) and (7.25). The proof is now completed by (7.26).

Theorem 7.1. Let $\delta_{n}=\left(\delta_{n, 1}, \delta_{n, 2}, \cdots, \delta_{n, n}\right)^{T}, f_{n}=\left(f_{n, 1}, f_{n, 2}, \cdots, f_{n, n}\right)^{T}$. If $\kappa>0$, then $A_{n} \delta_{n}=f_{n}$ has the unique solution $\delta_{n}=B_{n} f_{n}$ where $A_{n}$ and $B_{n}$ are the supplement matrices given in (7.13). If $\kappa<0$, then the condition of solvability for $A_{m, n} \delta_{n}=f_{m}$ is $B_{-2 \kappa, m} f_{m}=O_{-2 \kappa, 1}$ and it possesses a unique solution $\delta_{n}=$ $B_{n, m} f_{m}$ when the condition of solvability is fulfilled, where $A_{m, n}, B_{n, m}$ and $B_{-2 \kappa, m}$ are given in (7.2), (7.5) and (7.18), respectively. If $\kappa=0$ with $[\theta]_{\pi} \neq \pi / 2$, then $A_{n} \delta_{n}=f_{n}$ has the unique solution $\delta_{n}=B_{n} f_{n}$ where $A_{n}=A_{m, n}$ and $B_{n}$ are given in (7.2) and (7.22). If $\kappa=0$ with $[\theta]_{\pi}=\pi / 2$, then the condition of solvability for $A_{n+1, n} \delta_{n}=f_{n+1}$ is $B_{1, n+1} f_{n+1}=0$ and it possesses a unique solution $\delta_{n}=$ $B_{n, n+1} f_{n+1}$ when the condition of solvability is fulfilled, where $A_{n+1, n}, B_{n, n+1}$ and $B_{1, n+1}$ are given in (7.24) and (7.25), respectively.

Proof. (I) The proofs for the cases of $\kappa>0$ and $\kappa=0$ with $[\theta]_{\pi} \neq \frac{\pi}{2}$ are obvious by using Lemma 7.2 and Lemma 7.4. (II) For the case of $\kappa<0$, if $A_{m, n} \delta_{n}=f_{m}$, then, using, respectively, $B_{n, m}$ and $B_{-2 \kappa, m}$, by Lemma 7.3 we get $\delta_{n}=B_{n, m} f_{m}$ and $B_{-2 \kappa, m} f_{m}=O_{-2 \kappa, n} \delta_{n}=O_{-2 \kappa, 1}$. Conversely, if $B_{-2 \kappa, m} f_{m}=O_{-2 \kappa, 1}$, let $\delta_{n}=$ $B_{n, m} f_{m}$, then, by Lemma 7.3 we get $A_{m, n} \delta_{n}=A_{m, n} B_{n, m} f_{m}+A_{m,-2 \kappa} B_{-2 \kappa, m} f_{m}=$ $A_{m} B_{m} f_{m}=f_{m}$ where $A_{m,-2 \kappa}$ is given in (7.20). (III) The proof for the case of $\kappa=0$ with $[\theta]_{\pi}=\pi / 2$ is similar to (II).

\section{Direct QUADRATURE METHOD}

In this section, we introduce the direct quadrature method of SIE (2.8) rewritten in the form

$$
(\mathbf{A}+\lambda \mathbf{K}) y=f \quad \text { with } \quad(\mathbf{K} y)(t)=\frac{1}{2 \pi} \int_{0}^{2 \pi} w_{1}(\tau) k(\tau, t) y(\tau) \mathrm{d} \tau .
$$

From Remark 6.2 we know that, in general, $\mathbf{Q}_{m}^{\nabla B}$ is not defined if $f \in H_{2 \pi}$. This is not convenient in applications, therefore we must firstly improve $\mathbf{Q}_{m}^{\nabla B}$. 
We introduce the interpolation operator $\mathbf{L}_{m}^{\nabla}\left(f \mapsto \mathbf{L}_{m}^{\nabla} f\right)$, which possesses the interpolation property and differentiability at the zeros of $\nabla m$,

$$
\mathbf{r}_{m}^{\nabla} \mathbf{L}_{m}^{\nabla} f=\mathbf{r}_{m}^{\nabla} f, \quad \mathbf{L}_{m}^{\nabla} f \in C_{2 \pi}^{\prime}(\nabla m) .
$$

For example, the trigonometric interpolation polynomial operator $L_{m}^{\nabla}$ given by (6.5) is just one.

We introduce again the operator $\mathbf{Q}_{n}^{\triangle K}$ as

$$
\begin{aligned}
\left(\mathbf{Q}_{n}^{\triangle K} f\right)(t) & =E_{n}^{\triangle K}(t) \mathbf{r}_{n}^{\triangle} f \\
& \equiv\left(u_{n, 1}\left(\mathbf{L}_{m}^{\nabla} k_{\tau}\right)\left(\alpha_{n, 1}, t\right), \cdots, u_{n, n}\left(\mathbf{L}_{m}^{\nabla} k_{\tau}\right)\left(\alpha_{n, n}, t\right)\right) \mathbf{r}_{n}^{\triangle} f
\end{aligned}
$$

where

$$
\left(\mathbf{L}_{m}^{\nabla} k_{\tau}\right)(\tau, \cdot)=\left(\mathbf{L}_{m}^{\nabla} k_{\tau}\right)(\cdot),
$$

namely, applying $\mathbf{L}_{m}^{\nabla}$ to $k(\tau, t)$ for the second variable $t$ while the first variable $\tau$ is treated as a parameter we obtain the function $\mathbf{L}_{m}^{\nabla} k$ of two variables which is sometimes also denoted as $\left(\mathbf{L}_{m}^{\nabla} k\right)(\tau, t)$.

Remark 8.1. Obviously, Ima $\left(\mathbf{Q}_{n}^{\triangle K}\right) \subseteq C_{2 \pi}^{\prime}\left(\nabla_{m}\right)$.

Again applying the discretization operator $\mathbf{r}_{m}^{\nabla}$ to (8.3) we get

$$
\mathbf{r}_{m}^{\nabla} \mathbf{Q}_{n}^{\triangle K} f=K_{m, n} \mathbf{r}_{n}^{\triangle} f
$$

where the $(m, n)$ matrix

$$
K_{m, n}=\left(k_{i, j}\right) \text { with } k_{i, j}=u_{n, j} k\left(\alpha_{n, j}, \beta_{m, i}\right) .
$$

Now we introduce the direct quadrature method. We must separately consider four cases for the index $\kappa$.

The case of $\kappa>0$. In this case, by Theorem 5.3, in order that SIE (8.1) has a unique solution, we must further require that

$$
\mathbf{D}_{1} y=N_{\kappa} \in H_{\kappa}^{T}\left([\theta]_{\pi}\right) \text { (a given trigonometric polynomial). }
$$

In the system of equations (SE)

$$
\left\{\begin{array}{l}
(\mathbf{A}+\lambda \mathbf{K}) y=f \\
\mathbf{D}_{1} y=N_{\kappa}
\end{array}\right.
$$

replacing the operators $\mathbf{A}, \mathbf{K}, \mathbf{D}_{1}$ and $f$ with $\mathbf{Q}_{n}^{\triangle A}, \mathbf{Q}_{n}^{\triangle K}, \mathbf{Q}_{n}^{\triangle D_{1}}$ and $\mathbf{L}_{m}^{\nabla} f$ respectively, we may construct the system of two functional equations

$$
\left\{\begin{array}{l}
\left(\mathbf{Q}_{n}^{\triangle A}+\lambda \mathbf{Q}_{n}^{\triangle K}\right) y_{n}=\mathbf{L}_{m}^{\nabla} f, \\
\mathbf{Q}_{n}^{\triangle D_{1}} y_{n}=N_{\kappa},
\end{array}\right.
$$

which is called the (direct) approximate equation (AE) of SE (8.8). Its solutions are called the (direct) approximate solutions of SE (8.8). Discretizing the first equation by $\mathbf{r}_{m}^{\nabla}$ and the second equation in (8.9) by $\mathbf{r}_{2 \kappa}^{\vee}$, we get a system of linear algebraic equations, which is called the (direct) numerical equation (NE) of $\mathrm{AE}(8.9)$ and $\mathrm{SE}$ (8.8),

$$
\left(A_{n}+\lambda K_{n}\right) \delta_{n}=f_{n},
$$

where $A_{n}$ is given in (7.13) and

$$
K_{n}=\left(\begin{array}{c}
K_{m, n} \\
O_{\kappa, n}
\end{array}\right), \quad f_{n}=\left(\begin{array}{c}
\mathbf{r}_{m}^{\nabla} f \\
\mathbf{r}_{2 \kappa}^{\vee} N_{\kappa}
\end{array}\right), \quad \delta_{n}=\left(\delta_{n, 1}, \delta_{n, 2}, \cdots, \delta_{n, n}\right)^{T},
$$


where $K_{m, n}$ is given in (8.6) and $O_{\kappa, n}$ is the $(k, n)$ zero matrix. The solutions of $\mathrm{NE}$ (8.10) are called the (direct) numerical solutions of AE (8.9) and SE (8.8).

We point out that there is a very interesting relation between the approximate solution and the numerical solution. Obviously, if $y_{n}$ is an approximate solution, then $\delta_{n}=\mathbf{r}_{n}^{\triangle} y_{n}$ is just a numerical solution. Contrarily, if $\delta_{n}$ is a numerical solution, we construct the extension operator

$$
\left(\mathbf{E}_{n}^{\triangle} \delta_{n}\right)(t)=\frac{\triangle_{n}(t)}{\nabla m(t)}\left[\left(\mathbf{L}_{m}^{\nabla} f\right)(t)-b(t) E_{n}^{\triangle A}(t) \delta_{n}-\lambda E_{n}^{\triangle K}(t) \delta_{n}\right] .
$$

Since $\delta_{n}$ is a numerical solution, the above $\mathbf{E}_{n}^{\triangle} \delta_{n}$ is exactly well defined and $\mathbf{E}_{n}^{\triangle} \delta_{n} \in$ $C_{2 \pi}^{\prime}\left(\triangle_{n}\right)$ by $(8.2)$ and Remark 6.1. Moreover, we may show that it possesses the interpolation property

$$
\mathbf{r}_{n}^{\triangle} \mathbf{E}_{n}^{\triangle} \delta_{n}=\delta_{n}
$$

To prove this, let $\delta$ denote a function which possesses $\mathbf{r}_{n}^{\triangle} \delta=\delta_{n}$, for example, the trigonometric interpolation polynomial. Then we have

$$
\mathbf{r}_{n}^{\triangle} \mathbf{E}_{n}^{\triangle} \delta_{n}=\mathbf{r}_{n}^{\triangle}\left\{\delta+\frac{\triangle_{n}}{\nabla_{m}}\left[\mathbf{L}_{m}^{\nabla} f-\left(\mathbf{Q}_{n}^{\triangle A}+\lambda \mathbf{Q}_{n}^{\triangle K}\right) \delta\right]\right\}=\mathbf{r}_{n}^{\triangle} \delta=\delta_{n}
$$

by $(8.12)$, since $\delta_{n}$ is a numerical solution.

Remark 8.2. Now the first equation in (8.9) may be rewritten as

$$
y_{n}=\mathbf{E}_{n}^{\triangle} \mathbf{r}_{n}^{\triangle} y_{n} .
$$

Therefore, by (8.13) $\mathbf{E}_{n}^{\triangle} \delta_{n}$ satisfies the first equation in (8.9) if $\delta_{n}$ is a numerical solution.

Remark 8.3. Noting (7.9) and (8.13), we know $\mathbf{r}_{2 \kappa}^{\vee} \mathbf{Q}_{n}^{\triangle D_{1}} \mathbf{E}_{n}^{\triangle} \delta_{n}=A_{2 \kappa, n} \mathbf{r}_{n}^{\triangle} \mathbf{E}_{n}^{\triangle} \delta_{n}=$ $A_{2 \kappa, n} \delta_{n}=\mathbf{r}_{2 \kappa}^{\vee} N_{\kappa}$. Again, by Corollary 5.1, $\mathbf{Q}_{n}^{\triangle D_{1}} \mathbf{E}_{n}^{\triangle} \delta_{n} \in H_{\kappa}^{T}\left([\theta]_{\pi}\right)$; finally, by the uniqueness of the trigonometric interpolation on $H_{\kappa}^{T}\left([\theta]_{\pi}\right)$ [24, 26], we get $\mathbf{Q}_{n}^{\triangle D_{1}} \mathbf{E}_{n}^{\triangle} \delta_{n}=N_{\kappa}$, i.e., $\mathbf{E}_{n}^{\triangle} \delta_{n}$ satisfies the second equation in (8.9).

Combining Remark 8.2 and Remark 8.3, we know $E_{n}^{\triangle} \delta_{n}$ is an approximate solution. Thus, we may solve AE (8.9) via solving NE (8.10). More precisely, noting (8.13) and (8.14) we have the following theorem.

Theorem 8.1. Let $F^{D}$ be the set of the (direct) approximate solutions and $E^{D}$ the set of the (direct) numerical solutions, then the extension operator $\mathbf{E}_{n}^{\triangle}: E^{D} \rightarrow F^{D}$ given in (8.12) and the discretization operator $\mathbf{r}_{n}^{\triangle}: F^{D} \rightarrow E^{D}$ are inverse to each other.

The case of $\kappa<0$. In this case, prescribing that (8.1) has a solution, by Theorem 5.3 we see that the following constraint condition must be satisfied:

$$
\mathbf{D}_{2}[f-\lambda \mathbf{K} y]=0 .
$$

Now we cannot directly construct the first functional equation in (8.9), because it is difficult to ensure its solvability when $\kappa<0$. In fact, by Theorem 6.2 if it has a solution $y_{n}$, then necessarily

$$
\mathbf{Q}_{n}^{\triangle D_{2}} g^{*}=0 \text { with } g^{*}=\mathbf{L}_{m}^{\nabla} f-\lambda \mathbf{Q}_{n}^{\triangle K} y_{n} .
$$


In general, (8.16) does not follow from (8.15). A reasonable method is to consider both (8.1) and the constraint condition (8.15). It may be seen that the solution of (8.1) must be the solution of the equation

$$
(A+\lambda \mathbf{K}) y=f+b \mathbf{D}_{2}(f-\lambda \mathbf{K} y) .
$$

In this equation, replacing the operators $\mathbf{A}, \mathbf{K}, \mathbf{D}_{2}, f$ with $\mathbf{Q}_{n}^{\triangle A}, \mathbf{Q}_{n}^{\triangle K}, \mathbf{Q}_{m}^{\nabla D_{2}}$, $\mathbf{L}_{m}^{\nabla} f$, respectively, we construct the functional equation

$$
\begin{aligned}
\left(\mathbf{Q}_{n}^{\triangle A}+\lambda \mathbf{Q}_{n}^{\triangle K}+\lambda b \mathbf{Q}_{m}^{\nabla D_{2}} \mathbf{Q}_{n}^{\triangle K}\right) y_{n}=f^{*} & \\
& =\mathbf{L}_{m}^{\nabla} f+b N_{-\kappa} \quad\left(N_{-\kappa}=\mathbf{Q}_{m}^{\nabla D_{2}} f \in H_{-\kappa}^{T}\left([-\theta]_{\pi}\right)\right) .
\end{aligned}
$$

Discretizing (8.18) by $\mathbf{r}_{m}^{\nabla}$, we get the system of linear algebraic equations

$$
\left(A_{m, n}+\lambda K_{m, n}+\lambda K_{m, n}^{*}\right) \delta_{n}=f_{m}^{*}=\mathbf{r}_{m}^{\nabla} f^{*},
$$

where $A_{m, n}, K_{m, n}$ are as before, and the $(m, n)$ matrix

$$
\begin{gathered}
K_{m, n}^{*}=\left(k_{i, j}^{*}\right), k_{i, j}^{*}=b\left(\beta_{m, i}\right) u_{n, j} k_{-\kappa}^{j}\left(\beta_{m, i}\right), \\
k_{-\kappa}^{j}=\mathbf{Q}_{m}^{\nabla D_{2}} k^{j} \in H_{-\kappa}^{T}\left([-\theta]_{\pi}\right), k^{j}=k\left(\alpha_{n, j}, t\right) .
\end{gathered}
$$

Remark 8.4. $K_{m, n}^{*} \mathbf{r}_{n}^{\triangle} f=\mathbf{r}_{m}^{\nabla}\left(b \mathbf{Q}_{m}^{\nabla D_{2}} \mathbf{Q}_{n}^{\triangle K}\right) f$.

Now, we call (8.18) and (8.19) the (direct) approximate equation (AE) and the (direct) numerical equation (NE) of (8.17), respectively. Their solutions are called the (direct) approximate solution and the (direct) numerical solution of (8.1), respectively. If $\delta_{n}$ is a numerical solution, by setting the extension operator

$$
\left(\mathbf{E}_{n}^{\triangle} \delta_{n}\right)(t)=\frac{\triangle_{n}(t)}{\nabla_{m}(t)}\left\{f^{*}(t)-\left[b(t) E_{n}^{\triangle A}(t)+\lambda E_{n}^{\triangle K}(t)+\lambda b(t) E_{n}^{\triangle K^{*}}(t)\right] \delta_{n}\right\}
$$

where

$$
\begin{array}{r}
E_{n}^{\triangle K^{*}}(t)=\left(u_{n, 1} k^{*}\left(\alpha_{n, 1}, t\right), u_{n, 2} k^{*}\left(\alpha_{n, 2}, t\right), \cdots, u_{n, n} k^{*}\left(\alpha_{n, n}, t\right)\right) \\
\text { with } k^{*}(\tau, t)=\left(\mathbf{Q}_{m}^{\nabla D_{2}} \mathbf{L}_{m}^{\nabla} k_{\tau}\right)(\tau, t)
\end{array}
$$

in which the first variable $\tau$ is always treated as a parameter, then Theorem 8.1 still holds and the proof follows from working in a way analogous to that in the case $\kappa>0$.

The case of $\kappa=0$ with $[\theta]_{\pi}=\pi / 2$. Assume (8.1) has a solution, then the constraint condition, by Theorem 5.3 and (5.9),

$$
\mathbf{V} g=0 \text { with } g=f-\lambda \mathbf{K} y,
$$

must be satisfied; moreover, in order to find the determined solution we need the unisolving condition

$$
\mathbf{U} y=-N \csc \theta \quad(N \text { is a given constant and } \csc \theta= \pm 1) .
$$

For the discussion of the numerical solution under the present case, we must keep an eye on both the constraint condition (8.23) and the unisolving condition (8.24). Obviously, the solution of (8.1) is the solution of the equation (8.17). As before, from

$$
\left\{\begin{array}{l}
(A+\lambda \mathbf{K}) y=f+b \mathbf{D}_{2}(f-\lambda \mathbf{K} y), \\
\mathbf{U} y=-N \csc \theta
\end{array}\right.
$$


we get the (direct) approximate equation $(\mathrm{AE})$

$$
\left\{\begin{array}{c}
\left(\mathbf{Q}_{n}^{\triangle A}+\lambda \mathbf{Q}_{n}^{\triangle K}+\lambda b \sin \theta \mathbf{Q}_{m}^{\nabla V} \mathbf{Q}_{n}^{\triangle K}\right) y_{n}=f^{*} \\
\quad \text { where }\left(\mathbf{L}_{m}^{\nabla} f\right)+b \sin \theta \mathbf{Q}_{m}^{\nabla V} f, \\
\mathbf{Q}_{n}^{\triangle U} y_{n}=-N \csc \theta
\end{array}\right.
$$

and the (direct) numerical equation $(\mathrm{NE})$

$$
\left(A_{n+1, n}+\lambda K_{n+1, n}+\lambda K_{n+1, n}^{*}\right) \delta_{n}=f_{n+1}^{*}
$$

where $A_{n+1, n}$ is given in (7.25),

$$
\begin{gathered}
K_{n+1, n}=\left(\begin{array}{c}
K_{m, n} \\
O_{1, n}
\end{array}\right), \quad K_{n+1, n}^{*}=\left(\begin{array}{c}
K_{m, n}^{*} \\
O_{1, n}
\end{array}\right), \\
f_{n+1}^{*}=\left(f^{*}\left(\beta_{m, 1}\right), \cdots, f^{*}\left(\beta_{m, m}\right),-N \csc \theta\right)^{T}
\end{gathered}
$$

with $K_{m, n}$ as (8.6) and $K_{m, n}^{*}$ as (8.20).

In the present case, we call the solutions of (8.26) and (8.27) the (direct) approximate solution and the (direct) numerical solution of (8.25), respectively. If we set the extension operator the same as (8.21), then Theorem 8.1 still holds.

The case of $\kappa=0$ with $[\theta]_{\pi} \neq \pi / 2$. Treating (8.1) as before, we obtain the functional equation and the linear algebraic equation

$$
\begin{gathered}
\left(\mathbf{Q}_{n}^{\triangle A}+\lambda \mathbf{Q}_{n}^{\triangle K}\right) y_{n}=\mathbf{L}_{m}^{\nabla} f, \\
\left(A_{n}+\lambda K_{n}\right) \delta_{n}=f_{n} \equiv\left(f\left(\beta_{m, 1}\right), f\left(\beta_{m, 2}\right), \cdots, f\left(\beta_{m, m}\right)\right)^{T}
\end{gathered}
$$

where $A_{n}=A_{m, n}$ and $K_{n}=K_{m, n}$ given in (7.2) and (8.6), respectively, are the square matrices since $m=n$.

As before, (8.29) and (8.30) are called, respectively, the (direct) approximate equation (AE) and the (direct) numerical equation (NE) of (8.1). Their solutions are called the (direct) approximate solution and the (direct) numerical solution of (8.1), respectively. If $\delta_{n}$ is a numerical solution, by still setting the extension operator $\mathbf{E}_{n}^{\triangle}$ as (8.12), then Theorem 8.1 holds again.

\section{INDIRECT QUADRATURE METHOD}

In the present section, we discuss the indirect quadrature method. When $\kappa>0$, applying $\mathbf{B}$ to both sides of (8.1) and taking into account the unisolving condition (8.7), (8.8) becomes as, by Theorem 5.3 ,

$$
(\mathbf{I}+\lambda \mathbf{L}) y=F \equiv \mathbf{B} f+b N_{\kappa}
$$

$$
\text { with }(\mathbf{L} y)(t)=(\mathbf{B K} y)(t)=\frac{1}{2 \pi} \int_{0}^{2 \pi} w_{1}(\tau) l(\tau, t) y(\tau) d \tau
$$

where

$$
\begin{aligned}
l(\tau, t)= & \left(\mathbf{B} k_{\tau}\right)(t)=(\mathbf{B} k)(\tau, t) \\
& \equiv a(t) w_{2}(t) k(\tau, t)-\frac{b(t)}{2 \pi} \int_{0}^{2 \pi} w_{2}(x) k(\tau, x) \cot \frac{x-t}{2} \mathrm{~d} x .
\end{aligned}
$$

In the above $\mathbf{B} k_{\tau}$ we also treat $\tau$ as a parameter.

In the case of $\kappa<0$, noting Theorem 5.2, Remark 5.4 and Corollary 5.2, applying B to (8.17) we again obtain (9.1), but we must treat $N_{\kappa}=0$ in (9.1) (this is just in agreement with what we agreed before). 
Similarly, in the case of $\kappa=0$ with $[\theta]_{\pi}=\pi / 2$, applying $\mathbf{B}$ to both sides of (8.17) and taking into account the unisolving condition (8.24), by using Theorem 5.3, Remark 5.4 and Corollary 5.2, we still obtain (9.1) by treating $N_{0}=N$ given in (8.24).

In the case of $\kappa=0$ with $[\theta]_{\pi} \neq \pi / 2$, applying $\mathbf{B}-b \sec \theta \mathbf{D}_{2}$ to both sides of (8.1), by Remark 5.5 we also obtain (9.1) with the understanding of

$$
l(\tau, t)=\left(\mathbf{B} k_{\tau}\right)(\tau, t)-\frac{b(t) \tan \theta}{2 \pi} \int_{0}^{2 \pi} w_{2}(t) k(\tau, t) \mathrm{d} t, \quad N_{0}=-\tan \theta \mathbf{V} f .
$$

We call the Fredholm integral equation (9.1) the regularizing equation, respectively, of (8.8) when $\kappa>0$, of (8.17) when $\kappa<0$, of (8.25) when $\kappa=0$ with $[\theta]_{\pi}=\pi / 2$, of $(8.1)$ when $\kappa=0$ with $[\theta]_{\pi} \neq \pi / 2$, with the understanding for $l$ and $N_{\kappa}$ in (9.1) and (9.2),

$$
\begin{aligned}
& l= \begin{cases}l \text { given in }(9.2), & \text { when } \kappa \neq 0 \text { or } \kappa=0 \text { with }[\theta]_{\pi}=\pi / 2, \\
l \text { given in }(9.3), & \text { when } \kappa=0 \text { with }[\theta]_{\pi}=\pi / 2,\end{cases} \\
& N_{\kappa}= \begin{cases}N_{\kappa} \text { given in }(8.7), & \text { when } \kappa>0, \\
0, & \text { when } \kappa<0, \\
N \text { given in }(8.24), & \text { when } \kappa=0 \text { with }[\theta]_{\pi}=\pi / 2, \\
-\tan \theta \mathbf{V} f, & \text { when } \kappa=0 \text { with }[\theta]_{\pi} \neq \pi / 2 .\end{cases}
\end{aligned}
$$

In this way, the discussion below shall be valid for all cases of the index $\kappa$.

Remark 9.1. From Remark 5.2, $F$ and $l$ are functions in $H_{2 \pi}$ for all cases of $\kappa$.

Instead of (9.2), we use the operator

$$
\begin{aligned}
\left(\mathbf{Q}_{n}^{\triangle L} y\right)(t) & =E_{n}^{\triangle L}(t) \mathbf{r}_{n}^{\triangle} y \\
& \equiv\left(u_{n, 1} l\left(\alpha_{n, 1}, t\right), u_{n, 2} l\left(\alpha_{n, 2}, t\right), \cdots, u_{n, n} l\left(\alpha_{n, n}, t\right)\right) \mathbf{r}_{n}^{\triangle} y,
\end{aligned}
$$

then (9.1) becomes

$$
\left(\mathbf{I}+\lambda \mathbf{Q}_{n}^{\triangle L}\right) y=F
$$

Again, setting

$$
l_{n}(\tau, t)=\left\{\begin{aligned}
\left(\mathbf{Q}_{m}^{\nabla B} \mathbf{L}_{m}^{\nabla} k_{\tau}\right)(\tau, t)-b(t) \tan \theta\left(\mathbf{Q}_{m}^{\nabla V} \mathbf{L}_{m}^{\nabla} k_{\tau}\right)(\tau), & \text { if } \kappa=0 \text { with }[\theta]_{\pi} \neq \pi / 2, \\
\left(\mathbf{Q}_{m}^{\nabla B} \mathbf{L}_{m}^{\nabla} k_{\tau}\right)(\tau, t), & \text { otherwise, }
\end{aligned}\right.
$$

where

$$
\begin{aligned}
\left(\mathbf{Q}_{m}^{\nabla B} \mathbf{L}_{m}^{\nabla} k_{\tau}\right)(\tau, t)= & \frac{\triangle_{n}(t)}{\nabla m(t)}\left(\mathbf{L}_{m}^{\nabla} k_{\tau}\right)(\tau, t) \\
& -b(t) \sum_{j=1}^{m} v_{m, j} \cot \frac{v_{m, j}-t}{2}\left(\mathbf{L}_{m}^{\nabla} k_{\tau}\right)\left(\tau, v_{m, j}\right), \\
\left(\mathbf{Q}_{m}^{\nabla V} \mathbf{L}_{m}^{\nabla} k_{\tau}\right)(\tau)= & \mathbf{Q}_{m}^{\nabla V} \mathbf{L}_{m}^{\nabla} k_{\tau}=\sum_{j=1}^{m} v_{m, j}\left(\mathbf{L}_{m}^{\nabla} k_{\tau}\right)\left(\tau, v_{m, j}\right) .
\end{aligned}
$$

In (9.6) replacing $l$ by $l_{n}$, we have

$$
\begin{aligned}
\left(\mathbf{Q}_{n}^{\triangle L_{n}} y\right)(t) & =E_{n}^{\triangle L_{n}}(t) \mathbf{r}_{n}^{\triangle} y \\
& \equiv\left(u_{n, 1} l_{n}\left(\alpha_{n, 1}, t\right), u_{n, 2} l_{n}\left(\alpha_{n, 2}, t\right), \cdots, u_{n, n} l_{n}\left(\alpha_{n, n}, t\right)\right) \mathbf{r}_{n}^{\triangle} y
\end{aligned}
$$


Remark 9.2. We easily see $l_{n}(\tau, \cdot) \in C_{2 \pi}^{\prime}\left(\triangle_{n}\right)$ by Remark 6.2, so $\operatorname{Ima}\left(\mathbf{Q}_{n}^{\triangle L_{n}}\right) \subseteq$ $C_{2 \pi}^{\prime}\left(\triangle_{n}\right)$.

Remark 9.3. From (8.3), (9.9) and (9.10) we get $\mathbf{Q}_{n}^{\triangle L_{n}}=\left[\mathbf{Q}_{m}^{\nabla B}-b \tan \theta \mathbf{Q}_{m}^{\nabla V}\right] \mathbf{Q}_{n}^{\triangle K}$, while $\kappa=0$ with $[\theta]_{\pi} \neq \pi / 2$ and $\mathbf{Q}_{n}^{\triangle L_{n}}=\mathbf{Q}_{m}^{\nabla B} \mathbf{Q}_{n}^{\triangle K}$, while $\kappa \neq 0$ or $\kappa=0$ with $[\theta]_{\pi}=\pi / 2$.

Remark 9.4. If $\kappa \neq 0$ or $\kappa=0$ with $[\theta]_{\pi}=\pi / 2$, applying $\mathbf{Q}_{n}^{\triangle A}$ and $\mathbf{Q}_{n}^{\triangle D_{1}}$ to $\mathbf{Q}_{n}^{\triangle L_{n}}=\mathbf{Q}_{m}^{\nabla B} \mathbf{Q}_{n}^{\triangle K}$, we get $\mathbf{Q}_{n}^{\triangle A} \mathbf{Q}_{n}^{\triangle L_{n}}=\mathbf{Q}_{n}^{\triangle K}+b \mathbf{Q}_{m}^{\nabla D_{2}} \mathbf{Q}_{n}^{\triangle K}$ and $\mathbf{Q}_{n}^{\triangle D_{1}} \mathbf{Q}_{n}^{\triangle L_{n}}=0$ by Theorem 6.2 and Corollary 6.2.

Now we construct the functional equation

$$
\left(\mathbf{I}+\lambda \mathbf{Q}_{n}^{\triangle L_{n}}\right) y_{n}=F_{n} \equiv \mathbf{Q}_{m}^{\nabla B} \mathbf{L}_{m}^{\nabla} f+b N_{\kappa}\left(N_{\kappa} \text { in }(9.5)\right) .
$$

(9.11) is called the approximate equation (AE) of (9.1) or, respectively, the (indirect) approximate equation of (8.8) under the case $\kappa>0$, of (8.17) under the case $\kappa<0$, of (8.25) under the case $\kappa=0$ with $[\theta]_{\pi}=\pi / 2$, of (8.1) under the case $\kappa=0$ with $[\theta]_{\pi} \neq \pi / 2$. Its solutions are called the (indirect) approximate solutions, the set of which is written as $F^{I}$.

Remark 9.5. $F^{I} \subseteq C_{2 \pi}^{\prime}\left(\triangle_{n}\right)$, namely, in essence we find the approximate solutions in $C_{2 \pi}^{\prime}\left(\triangle_{n}\right)$.

Discretizing (9.11) by $\mathbf{r}_{n}^{\triangle}$, we get a system of linear algebraic equations as

$$
\left(I_{n}+\lambda L_{n}\right) \delta_{n}=\mathbf{r}_{n}^{\triangle} F_{n},
$$

where the square matrix of order $n$ is

$$
L_{n}=\left(l_{j, r}\right) \text { with } l_{j, r}=u_{n, r} l_{n}\left(\alpha_{n, r}, \alpha_{n, j}\right) .
$$

Remark 9.6. $\mathbf{r}_{n}^{\triangle} \mathbf{Q}_{n}^{\triangle L_{n}}=L_{n} \mathbf{r}_{n}^{\triangle}$.

(9.12) is called the numerical equation (NE) of $\mathrm{AE} \mathrm{(9.11),} \mathrm{or} \mathrm{respectively,} \mathrm{the}$ (indirect) numerical equation of (8.8) under the case $\kappa>0$, of (8.17) under the case $\kappa<0$, of $(8.25)$ under the case $\kappa=0$ with $[\theta]_{\pi}=\pi / 2$, of (8.1) under the case $\kappa=0$ with $[\theta]_{\pi} \neq \pi / 2$. Its solutions are called (indirect) numerical solutions. $E^{I}$ denotes the set of the (indirect) numerical solutions.

Now working in a way analogous to that used in the last section, we can easily show that, between the (indirect) approximate solutions and the (indirect) numerical solutions there exists the following relation.

Theorem 9.1. Either both the (indirect) approximate solutions and the (indirect) numerical solutions exist or neither exists. If they exist, then they are in one-to-one correspondence: $\mathbf{r}_{n}^{\triangle}$ being a one-to-one operator for $F^{I} \rightarrow E^{I}, \mathbf{E}_{n}^{\triangle}$ being a one-toone operator for $E^{I} \rightarrow F^{I}$ and they are inverse to each other, where the operator $\mathbf{E}_{n}^{\triangle}$ is an extension operator as follows:

$$
\left(\mathbf{E}_{n}^{\triangle} \delta_{n}\right)(t)=F_{n}(t)-\lambda E_{n}^{\triangle L_{n}}(t) \delta_{n} \quad \text { if } \quad \delta_{n} \in E^{I} .
$$

In the direct quadrature method and indirect quadrature method, we only require that the interpolation operator $\mathbf{L}_{m}^{\nabla}$ satisfies (8.2). Therefore, we may choose the $\mathbf{L}_{m}^{\nabla}$ according to the input condition and the requirement of the problem. There are two useful choices of $\mathbf{L}_{m}^{\nabla}$ (the other will be introduced in another paper). If the input functions $f, k \in C_{2 \pi}^{\prime}$, for the higher trigonometric precision, sometimes one chooses $\mathbf{L}_{m}^{\nabla}=\mathbf{I}$ (the identity operator). For the general case that the input functions $f, k$ 
are in the class $H_{2 \pi}$, we take $\mathbf{L}_{m}^{\nabla}=L_{m}^{\nabla}$, the trigonometric interpolation polynomial operator given in (6.5).

Obviously, under any choice for $\mathbf{L}_{m}^{\nabla}$, the numerical solutions are always the same. We call the approximate solutions for the case $\mathbf{L}_{m}^{\nabla}=\mathbf{I}$ the approximate solutions of the first kind, i.e., Nyström's interpolation approximate solutions. For case $\mathbf{L}_{m}^{\nabla}=L_{m}^{\nabla}$, in Remark 10.2 of the next section we will see that the approximate solution is just the trigonometric interpolation polynomial at the zeros of $\triangle_{n}$ via the numerical solution, which is called the approximate solution of the second kind. In the framework given here, both the approximate solutions of the first and second kinds are the natural interpolation solutions via (8.12) or (8.21) or (9.14).

\section{Coincidence}

In the present section, we shall verify that the direct quadrature method and the indirect quadrature method stated above are equivalent. Due to this reason, we only need to discuss the existence and convergence of the (indirect) approximate solution and the (indirect) numerical solution. Such a coincidence technique is first studied by Ioakimidis [16] for the simplest case that $a$ and $b$ are constants. Here we discuss it in a quite simple and obvious way, so the results are more general and accurate.

Theorem 10.1. If $\kappa>0$, then (8.8) is equivalent to the regularizing equation (9.1). If $\kappa<0$, then (8.17) is equivalent to the regularizing equation (9.1). If $\kappa=0$ with $[\theta]_{\pi}=\pi / 2$, then (8.25) is equivalent to the regularizing equation (9.1). If $\kappa=0$ with $[\theta]_{\pi} \neq \pi / 2$, then (8.1) is equivalent to the regularizing equation (9.1).

Proof. These conclusions directly result from Theorem 5.3 and Corollaries 5.1 and 5.2. For example, when $\kappa<0$, let $f^{\#}=f-\lambda \mathbf{K} y+b \mathbf{D}_{2}(f-\lambda \mathbf{K} y)$ in (8.17), then $\mathbf{D}_{2} f^{\#}=0$ by Corollary 5.1, thus (8.17) has the solution $y$ if and only if $y$ is the solution of (9.1) by Theorem 5.3 and Corollary 5.2.

Theorem 10.2. If $\kappa>0$, then $A E$ (8.9) is equivalent to $A E$ (9.11). If $\kappa<0$, then $A E$ (8.18) is equivalent to $A E(9.11)$. If $\kappa=0$ with $[\theta]_{\pi}=\pi / 2$, then $A E(8.26)$ is equivalent to $A E$ (9.11). If $\kappa=0$ with $[\theta]_{\pi} \neq \pi / 2$, then $A E(8.29)$ is equivalent to $A E$ (9.11). In a word, $F^{D}=F^{I}$.

Proof. These conclusions directly result from Theorem 6.2 and Corollaries 6.1 and 6.2. For example, when $\kappa=0$ with $[\theta]_{\pi}=\pi / 2$, let

$$
f^{\#}=f^{*}-\left(\lambda \mathbf{Q}_{n}^{\triangle K}+\lambda b \sin \theta \mathbf{Q}_{m}^{\nabla V} \mathbf{Q}_{n}^{\triangle K}\right) y_{n}
$$

in (8.26), noting $\mathbf{Q}_{m}^{\nabla D_{2}}=b \sin \theta \mathbf{Q}_{m}^{\nabla V}$, then $\mathbf{Q}_{m}^{\nabla D_{2}} f^{\#}=0$ by Corollary 6.2. Thus (8.26) has the solution $y_{n}$ if and only if $y_{n}$ is the solution of (9.11) by using Theorem 6.2, Corollary 6.2 and Remark 9.4.

Remark 10.1. In passing, we point out an interesting fact. While $\kappa<0$, from Theorem 10.2 we see that, to solve $\mathrm{AE}(8.18)$ it does not need to know $N_{-\kappa}$ and $k_{-\kappa}^{j}$ introduced in (8.18) and (8.20), since they do not arise in (9.11). This shows that the method here is both complete in the theoretical analysis and convenient in the actual computation.

Theorem 10.3. If $\kappa>0$, then $N E$ (8.10) is equivalent to $N E$ (9.12). If $\kappa<0$, then $N E(8.19)$ is equivalent to $N E(9.12)$. If $\kappa=0$ with $[\theta]_{\pi}=\pi / 2$, then $N E(8.27)$ 
is equivalent to $N E$ (9.12). If $\kappa=0$ with $[\theta]_{\pi} \neq \pi / 2$, then $N E$ (8.30) is equivalent to $E N(9.12)$. In a word, $E^{D}=E^{I}$.

More precisely, we give some lemmas. These lemmas imply Theorem 10.3. For simplicity, we use some pictographic symbols, for example, let us denote to apply $B_{n}$ to both sides of (8.10) by $B_{n} \times(8.10)$.

Lemma 10.1. If $\kappa>0, A_{n}$ and $B_{n}$ are given in (7.13), then $B_{n} \times(8.10) \Rightarrow(9.12)$ and $A_{n} \times(9.12) \Rightarrow(8.10)$.

Proof. We prove the the first conclusion, since the second follows by it and Lemma 7.2. First, we have $L_{n} \mathbf{r}_{n}^{\triangle} y=\mathbf{r}_{n}^{\triangle} \mathbf{Q}_{n}^{\triangle L_{n}} y=\mathbf{r}_{n}^{\triangle} \mathbf{Q}_{m}^{\nabla B} \mathbf{Q}_{n}^{\triangle K} y=B_{n, m} \mathbf{r}_{m}^{\nabla} \mathbf{Q}_{n}^{\triangle K} y=$ $B_{n, m} K_{m, n} \mathbf{r}_{n}^{\triangle} y$, by Remark 9.6, Remark 9.3, (7.4) and (8.5), thus, $L_{n}=B_{n, m} K_{m, n}$. So, from (7.13) and (8.11) we get $L_{n}=B_{n} K_{n}$. Second, we get $\mathbf{r}_{n}^{\triangle} F_{n}=\mathbf{r}_{n}^{\triangleleft} \mathbf{Q}_{m}^{\nabla B} \mathbf{L}_{m}^{\nabla} f+$ $\mathbf{r}_{n}^{\triangle}\left(b N_{\kappa}\right)=B_{n, m} \mathbf{r}_{m}^{\nabla} f+\mathbf{r}_{n}^{\triangle}\left(b L_{2 \kappa}^{\vee} N_{\kappa}\right)=B_{n, m} \mathbf{r}_{m}^{\nabla} f+B_{n, 2 \kappa} \mathbf{r}_{2 \kappa}^{\vee} N_{\kappa}=B_{n} f_{n}$ by (9.11), (8.2), (7.11) and (8.11). So, $\mathbf{r}_{n}^{\triangle} F_{n}=B_{n} f_{n}$. Now, the proof is completed.

Lemma 10.2. If $\kappa<0, A_{m, n}$ and $B_{n, m}$ are given in (7.2) and (7.5), then $B_{n, m} \times$ $(8.19) \Rightarrow(9.12)$ and $A_{m, n} \times(9.12) \Rightarrow(8.19)$.

Proof. We prove the second conclusion, since the first one follows by it and Lemma 7.1. First,

$$
A_{m, n} L_{n} \mathbf{r}_{n}^{\triangle} y=A_{m, n} \mathbf{r}_{n}^{\triangle} \mathbf{Q}_{n}^{\triangle L_{n}} y=\mathbf{r}_{m}^{\nabla} \mathbf{Q}_{n}^{\triangle A} \mathbf{Q}_{n}^{\triangle L_{n}} y=\mathbf{r}_{m}^{\nabla}\left(\mathbf{Q}_{n}^{\triangle K} y+b \mathbf{Q}_{m}^{\nabla D_{2}} \mathbf{Q}_{n}^{\triangle K} y\right)
$$

by using Remark 9.6, (7.1), Remark 9.3 and (8.5), thus $A_{m, n} L_{n}=K_{m, n}+K_{m, n}^{*}$ from Remark 8.4. Second, by (9.11), (7.1), Theorem 6.4 and (8.18),

$$
A_{m, n} \mathbf{r}_{n}^{\triangle} F_{n}=\mathbf{r}_{m}^{\nabla} \mathbf{Q}_{n}^{\triangle A} F_{n}=\mathbf{r}_{m}^{\nabla} \mathbf{Q}_{n}^{\triangle A} \mathbf{Q}_{m}^{\nabla B} \mathbf{L}_{m}^{\nabla} f=\mathbf{r}_{m}^{\nabla}\left(\mathbf{L}_{m}^{\nabla} f+b \mathbf{Q}_{m}^{\nabla D_{2}} f\right)=\mathbf{r}_{m}^{\nabla} f_{m}^{*} .
$$

Lemma 10.3. If $\kappa=0$ with $[\theta]_{\pi}=\pi / 2$, then $B_{n, n+1} \times(8.27) \Rightarrow(9.12)$ and $A_{n+1, n} \times(9.12) \Rightarrow(8.27)$ where $A_{n, n+1}$ and $B_{n, n+1}$ are given in (7.24).

Proof. We prove the the second conclusion, since the first one follows by it and Lemma 7.5. By Remark 9.4, Remark 6.3, (6.10), and Remark 9.6 and noting $\sin \theta= \pm 1$, we have

$$
\begin{aligned}
0=\mathbf{Q}_{n}^{\triangle D_{1}} \mathbf{Q}_{n}^{\triangle L_{n}} y & = \pm \mathbf{Q}_{n}^{\triangle U} \mathbf{Q}_{n}^{\triangle L_{n}} y= \pm E_{n}^{\triangle U} \mathbf{r}_{n}^{\triangle} \mathbf{Q}_{n}^{\triangle L_{n}} y \\
& = \pm E_{n}^{\triangle U} L_{n} \mathbf{r}_{n}^{\triangle} y, \text { i.e., } E_{n}^{\triangle U} L_{n}=O_{1, n} .
\end{aligned}
$$

Noting that the equality $A_{m, n} L_{n}=K_{m, n}+K_{m, n}^{*}$ still holds in the present case in exactly the same way to the last lemma, and by (7.24) and (8.28), (10.1), we get

$$
A_{n+1, n} L_{n}=K_{n+1, n}+K_{n+1, n}^{*} .
$$

By (6.10) and (9.11), Remark 6.3, Corollaries 6.1 and 6.2, we have

$$
E_{n}^{\triangle U} \mathbf{r}_{n}^{\triangle} F_{n}=\mathbf{Q}_{n}^{\triangle U} F_{n}=-\csc \theta \mathbf{Q}_{n}^{\triangle D_{1}}\left(\mathbf{Q}_{m}^{\nabla B} \mathbf{L}_{m}^{\nabla} f+b N\right)=-N \csc \theta .
$$

Noting that the equality $A_{m, n} \mathbf{r}_{n}^{\triangle} F_{n}=\mathbf{r}_{m}^{\nabla} f^{*}$ still holds in the present case in exactly the same way to the last lemma and (7.24) and (8.28), we finally get

$$
A_{n+1, n} \mathbf{r}_{n}^{\triangle} F_{n}=f_{n+1}^{*} \text {. }
$$

Now, by (10.2) and (10.4), the second conclusion follows.

Lemma 10.4. If $\kappa=0$ with $[\theta]_{\pi} \neq \pi / 2$, then $B_{n} \times(8.30) \Rightarrow(9.12)$ and $A_{n} \times$ $(9.12) \Rightarrow(8.30)$ where $A_{n}$ and $B_{n}$ are given in (7.2) and (7.22), respectively. 
Proof. We prove the the second conclusion, since the first one follows by it and Lemma 7.4 in exactly the same way to Lemma 10.1. The equalities $B_{n} K_{n}=L_{n}$ and $B_{n} f_{n}=\mathbf{r}_{n}^{\triangle} F_{n}$ still hold in the present case. For example, $B_{n} f_{n}=B_{n} \mathbf{r}_{m}^{\nabla} \mathbf{L}_{m}^{\nabla} f=$ $\mathbf{r}_{n}^{\triangle}\left[\mathbf{Q}_{m}^{\nabla B}-b \tan \theta \mathbf{Q}_{m}^{\nabla V}\right] \mathbf{L}_{m}^{\nabla} f=\mathbf{r}_{n}^{\triangle} F_{n}$

Theorem 10.4. If $\kappa>0$ or $\kappa=0$ with $[\theta]_{\pi} \neq \pi / 2$, the extension operator defined by (8.12) and the extension operator defined by (9.14) are the same. If $\kappa<0$ or $\kappa=0$ with $[\theta]_{\pi}=\pi / 2$, the extension operator defined by (8.21) and the extension operator defined by (9.14) are the same.

Proof. By Theorem 8.1 (holds for any case), Theorem 9.1, Theorems 10.2 and 10.3, we know that all extension operators defined in $\S 7$ and $\S 8$ are the inverse operator of $\mathbf{r}_{n}^{\triangle}: F^{D} \rightarrow E^{D}$, so they are the same.

Remark 10.2. If $\mathbf{L}_{m}^{\nabla}=L_{m}^{\nabla}$ is the trigonometric interpolation operator given in (6.5), we may see that, $\mathbf{L}_{m}^{\nabla} f, E_{n}^{\triangle K}, f^{*}$ and $E_{n}^{\triangle K^{*}}$ in (8.12) and (8.21) are all trigonometric polynomials. From this and noting that $n-m=2 \kappa$ is even, we know that $\mathbf{E}_{n}^{\triangle} \delta_{n}$ given in both (8.12) and (8.21) are trigonometric polynomials of degree $\left[\frac{n}{2}\right]$ at most, so $\mathbf{E}_{n}^{\triangle} \delta_{n}$ is given in (9.14).

Some researchers studied the various coincidence theorems for a simple case such as (2.8) with the constants $a$ and $b$, but they all first proved $E^{D}=E^{I}$, which is based on Theorem 8.1 and obtained by very technical calculation, then they verify Theorem 10.4 and finally obtain the coincidence Theorem 10.2. This way is complex and difficult, and we can find the general law with difficulty. Under our framework the coincidence Theorems $10.2-10.4$ are obtained by applying the abstract properties of SIOs, SQOs and DMs associated with SIE (2.8) in a completely parallel way, hence can be applied more generally.

\section{Existence And CONVERGEnCE}

Suppose that there is a sequence of pairs $\left(\triangle_{n}, \nabla_{m}\right)$ of TPs of SIOs $(\mathbf{A}, \mathbf{B})$, say $\left\{\triangle_{n}, \nabla m\right\}$. We discuss now the existence and convergence of the approximate solution and the numerical solution. It suffices to discuss the existence and convergence of the indirect approximate solution by Theorems 10.2-10.4 and Theorem 9.1, provided that $\lambda$ is not an eigenvalue of (9.1), hence (9.1) possesses a unique solution. Therefore, by using Theorem 10.1, (8.8) also possesses a unique solution when $\kappa>0$ and (8.1) also possesses a unique solution when $\kappa=0$ with $[\theta]_{\pi} \neq \pi / 2$. When $\kappa<0,(8.17)$ possesses a unique solution but (8.1) has possibly no solution. In this case we further assume that (8.1) is solvable, thus it only possesses a unique solution. Similarly, when $\kappa=0$ with $[\theta]_{\pi}=\pi / 2$ we also assume that (8.1) is solvable, thus (8.1) under the unisolving condition (8.24) possesses a unique solution. We again suppose that the spaces considered are equipped with the Chebyshev norm $\|\cdot\|$, namely, the maximum of the absolute values of a function [34]. We will quote the theorems in [29, 30] with the same technical term there.

Lemma 11.1. Suppose that the sequence $\left\{\mathbf{Q}_{n}^{\triangle U}\right\}$ is uniformly bounded, then the sequence $\left\{\mathbf{Q}_{n}^{\triangle L}\right\}$ pointwisely approximates the operator $\mathbf{L}$, and $\left\{\mathbf{I}+\lambda \mathbf{Q}_{n}^{\triangle L}\right\}$ is stable.

Proof. By Remark 9.1 we may denote the modulus of continuity of $l(\tau, t) y(t)$ as $\omega(l y, \cdot)$. We then have $\left\|\left(\mathbf{L}-\mathbf{Q}_{n}^{\triangle L}\right) y\right\| \leq 12\left(\|\mathbf{U}\|+\left\|\mathbf{Q}_{n}^{\triangle U}\right\|\right) \omega(l y, 2 / n)$ by 
Theorem 5.1 in 30. Observing that $\left\{\mathbf{Q}_{n}^{\triangle U}\right\}$ is uniformly bounded and $\|\mathbf{U}\|=$ $\mathbf{U} 1$, thus, we know that the sequence $\left\{\mathbf{Q}_{n}^{\triangle L}\right\}$ pointwisely approximates the operator L. Again noting $\left\|\mathbf{Q}_{n}^{\triangle L}\right\| \leq\|l\|\left\|\mathbf{Q}_{n}^{\triangle U}\right\|, \quad\left|\left(\mathbf{Q}_{n}^{\triangle L} y\right)\left(t_{1}\right)-\left(\mathbf{Q}_{n}^{\triangle L} y\right)\left(t_{2}\right)\right| \leq$ $\|y\|\left\|\mathbf{Q}_{n}^{\triangle U}\right\| \omega\left(l,\left|t_{1}-t_{2}\right|\right)$ and the (uniform) continuity of $l$ by Remark 9.1, as well as using the Arzela-Ascoli lemma, we see that the $\left\{\mathbf{Q}_{n}^{\triangle L}\right\}$ is globally totally continuous. Therefore, $\left\{\mathbf{I}+\lambda \mathbf{Q}_{n}^{\triangle L}\right\}$ is stable by Theorem 2.3 in [30.

Theorem 11.1. Suppose (C1), the sequence $\left\{\mathbf{Q}_{n}^{\triangle U}\right\}$ is uniformly bounded, (C2) $\lim _{n \rightarrow \infty}\left\|F-F_{n}\right\|=0$ and $\lim _{n \rightarrow \infty}\left\|l-l_{n}\right\|=0$, then the numerical solution and the approximate solution uniquely exist for sufficiently large $n$, and the latter uniformly converges to the solution of SIE (9.1) with (9.4) and (9.5).

Proof. $\left\|\mathbf{Q}_{n}^{\triangle L}-\mathbf{Q}_{n}^{\triangle l_{n}}\right\| \leq\left\|\mathbf{Q}_{n}^{\triangle U}\right\|\left\|l-l_{n}\right\| \leq$ const $\left\|l-l_{n}\right\| \rightarrow 0($ as $n \rightarrow \infty)$, thereby, by further using Theorem 2.4 in [30] and Lemma 11.1 here, $\left\{\mathbf{I}+\lambda \mathbf{Q}_{n}^{\triangle L_{n}}\right\}$ is stable. By $(\mathrm{C} 2)$ and Theorem 2.5 in [30], the proof is completed.

We must demonstrate that $(\mathrm{C} 1)$ and $(\mathrm{C} 2)$ are satisfied when we use Theorem 11.1.

Remark 11.1. If $\left\{\mathbf{Q}_{n}^{\triangle U}\right\}$ is a sequence of nonnegative operators, i.e., $u_{n, j} \geq 0(j=$ $1, \cdots, n)$, then we have $\left\|\mathbf{Q}_{n}^{\triangle U}\right\|=\sum_{j=1}^{n} u_{n, j}=\mathbf{U} 1$; of course, it is uniformly bounded. The example will be given in the next section.

For (C2) in Theorem 11.1 we give two useful lemmas. From Theorem 5.2 in 27. we have the following.

Lemma 11.2. If $f \in C_{2 \pi}^{\prime}$ (i.e., $\left.f^{\prime} \in C_{2 \pi}\right)$, then

$$
\left\|\mathbf{B} f-\mathbf{Q}_{m}^{\nabla B} f\right\| \leq \text { const }\left(\|V\|+\left\|\mathbf{Q}_{m}^{\nabla V}\right\|\right) \omega\left(f^{\prime}, \frac{m}{2}\right) .
$$

In particular, If $\left\{\mathbf{Q}_{m}^{\nabla V}\right\}$ is uniformly bounded, then $\lim _{m \rightarrow \infty}\left\|\mathbf{B} f-\mathbf{Q}_{m}^{\nabla B} f\right\|=0$.

Lemma 11.3. If $f \in H_{2 \pi}^{\mu}$ (with the Hölder index $\mu$ in $\left.H_{2 \pi}\right), \operatorname{pr}\left(\mathbf{Q}_{m}^{\nabla B} \mathbf{L}_{m}^{\nabla}\right) \geq\left[\frac{m-1}{2}\right]$ and $\left\|\mathbf{Q}_{m}^{\nabla B} \mathbf{L}_{m}^{\nabla}\right\|=o\left(m^{\mu}\right)$, then $\lim _{m \rightarrow \infty}\left\|\mathbf{B} f-\mathbf{Q}_{m}^{\nabla B} \mathbf{L}_{m}^{\nabla} f\right\|=0$.

Proof. Denoting the best approximate trigonometric polynomial of degree not greater than $\nu=\left[\frac{m-1}{2}\right]$ of $f$ by $J_{\nu}$ and $f_{\nu}(\tau)=f(\tau)-J_{\nu}(\tau)$, then, by the Jackson theorem [31, 32, $\left\|f_{\nu}\right\| \leq 12 \omega\left(f, \frac{1}{\nu}\right)$, hence,

$$
\begin{aligned}
& \left|\left(\mathbf{B} f_{\nu}\right)(t)\right| \leq 12\|\mathbf{B} 1\| \omega\left(f, \frac{1}{\nu}\right)+\|b\|\left|\int_{-\pi+t}^{\pi+t} w_{2}(\tau) \frac{f_{\nu}(\tau)-f_{\nu}(t)}{\tau-t}\left[(\tau-t) \cot \frac{\tau-t}{2}\right] \mathrm{d} \tau\right| \\
& \leq 12\|\mathbf{B} 1\| \omega\left(f, \frac{1}{\nu}\right)+\frac{C\|w\|}{\nu \mu-\varepsilon} \int_{-\pi+t}^{\pi+t} \frac{d \tau}{|\tau-t|^{1-\varepsilon}}(0<\varepsilon<\mu)
\end{aligned}
$$

(by Lemma 5.2 and (5.5) in [27] or cf. [33], Hilfssatz 2, Sect. 2, Kapitel 2)

$$
\leq \frac{C_{1}\|\mathbf{B} 1\|}{\nu^{\mu}}+\frac{C_{2}\|w\|}{\varepsilon \nu^{\mu-\varepsilon}} \quad\left(C, C_{1}, C_{2} \text { are some constants only depending on } \mu \text { and } \varepsilon\right) \text {. }
$$


Again, $\left\|\left(\mathbf{Q}_{m}^{\nabla B} \mathbf{L}_{m}^{\nabla}\right) f_{\nu}\right\| \leq\left\|\mathbf{Q}_{m}^{\nabla B} \mathbf{L}_{m}^{\nabla}\right\|\left\|f_{\nu}\right\| \leq C_{3} \nu^{-\mu}\left\|\mathbf{Q}_{m}^{\nabla B} \mathbf{L}_{m}^{\nabla}\right\|$ where $C_{3}$ is also a constant only depending on $\mu$ and $\varepsilon$, and $\left[\mathbf{B}-\mathbf{Q}_{m}^{\nabla B} \mathbf{L}_{m}^{\nabla}\right] f=\left[\mathbf{B}-\mathbf{Q}_{m}^{\nabla^{B}} \mathbf{L}_{m}^{\nabla}\right] f_{\nu}$ by $\operatorname{pr}\left(\mathbf{Q}_{m}^{\nabla B} \mathbf{L}_{m}^{\nabla}\right) \geq \nu$. So, $\lim _{n \rightarrow \infty}\left\|\mathbf{B} f-\mathbf{Q}_{m}^{\nabla B} \mathbf{L}_{m}^{\nabla} f\right\|=0$.

Quoting the above lemmas and Theorem 11.1 we have the following two theorems for the existence and convergence of the approximate solutions.

Theorem 11.2. If $f \in C_{2 \pi}^{\prime}, k \in C_{2 \pi}^{\prime} \times C_{2 \pi}^{\prime}$ (i.e., $k$ has continuous partial derivatives), $\left\{\mathbf{Q}_{n}^{\triangle U}\right\}$ and $\left\{\mathbf{Q}_{m}^{\nabla V}\right\}$ are uniformly bounded, then the approximate solution of the first kind exists uniquely and converges uniformly to the solution of SIE (9.1) with (9.4) and (9.5).

Theorem 11.3. If $f, k \in H_{2 \pi}^{\mu},\left\{\mathbf{Q}_{n}^{\triangle U}\right\}$ is uniformly bounded and $\left\|\mathbf{Q}_{m}^{\nabla B} \mathbf{L}_{m}^{\nabla}\right\|=$ $o\left(m^{\mu}\right)$, then the approximate solution of the second kind exists uniquely and converges uniformly to the solution of SIE (9.1) with (9.4) and (9.5) while $\kappa \neq 0$ or $\kappa=0$ with $[\theta]_{\pi}=\pi / 2$. If $\kappa=0$ with $[\theta]_{\pi} \neq \pi / 2$, further assume $\left\|\mathbf{Q}_{m}^{\nabla V}\right\|=o\left(m^{\mu}\right)$, then the approximate solutions of the second kind exist uniquely and converge uniformly to the solution of SIE (8.1).

Remark 11.2. How to check $\left\|\mathbf{Q}_{m}^{\nabla B} \mathbf{L}_{m}^{\nabla}\right\|=\mathrm{o}\left(m^{\nu}\right)$, more precisely, how to estimate the order of $\left\|\mathbf{Q}_{m}^{\nabla B} \mathbf{L}_{m}^{\nabla}\right\|$. This problem is rather difficult, it will be studied in another forthcoming paper.

If we take $\mathbf{L}_{m}^{\nabla}$ as the Lagrange interpolating polynomial operator $L_{m}^{\nabla}$, let

$$
\nabla m, j(\tau)= \begin{cases}\frac{\nabla_{m}(\tau)}{2 \nabla_{m}^{\prime}\left(\beta_{m, j}\right)} \csc \frac{\tau-\beta_{m, j}}{2}, & \text { if } m \text { is odd } \\ \frac{\nabla_{m}(\tau)}{2 \nabla_{m}^{\prime}\left(\beta_{m, j}\right)} \cot \frac{\tau-\beta_{m, j}}{2}, & \text { if } m \text { is even }\end{cases}
$$

Then

$$
\begin{aligned}
& \left|\left(\mathbf{Q}_{m}^{\nabla B} L_{m}^{\nabla} f\right)(t)\right| \\
& =\left|\sum_{j=1}^{m}\left[a(t) w_{1}(t) \nabla_{m, j}(t)-\frac{b(t)}{2 \pi} \int_{0}^{2 \pi} \nabla_{m, j}(\tau) \cot \frac{\tau-t}{2} \mathrm{~d} \tau\right] f\left(\beta_{m, j}\right)\right| \\
& \leq\left\|a w_{1}\right\| \Sigma_{m}+\|b\| \sigma_{m},
\end{aligned}
$$

where

$$
\begin{gathered}
\left.\Sigma_{m}=\sum_{j=1}^{m}\|\nabla m, j\| \text { (the Lebesgue number of } L_{m}^{\nabla}\right), \\
\sigma_{m}=\sum_{j=1}^{m}\left\|\frac{1}{2 \pi} \int_{0}^{2 \pi} w_{2}(\tau) \nabla_{m, j}(\tau) \frac{\tau-t}{2} \mathrm{~d} \tau\right\| .
\end{gathered}
$$

Again, we have by Remark 6.1 that

$$
\left(\mathbf{Q}_{m}^{\nabla B} L_{m}^{\nabla} f\right)(t)=\sum_{j=1}^{m}\left\{\Lambda_{m, j}(t)+\left[b(t)-b\left(\beta_{m, j}\right)\right] \cot \frac{t-\beta_{m, j}}{2} v_{m, j}\right\} f\left(\beta_{m, j}\right)
$$


where

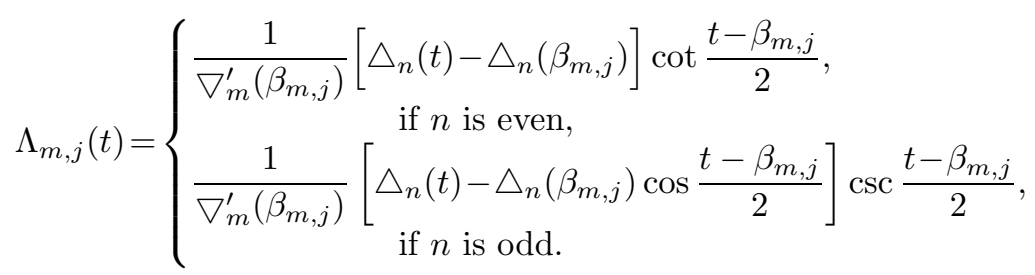

So, we also get

$$
\left\|\mathbf{Q}_{m}^{\nabla B} L_{m}^{\nabla}\right\| \leq \rho_{m}+2\left\|b^{\prime}\right\| \lambda_{m}, \rho_{m}=\sum_{j=1}^{m}\left\|\Lambda_{m, j}\right\|, \lambda_{m}=\sum_{j=1}^{m}\left|v_{m, j}\right| .
$$

For some $\{\nabla m\}$, used often, we easily estimate $\left\|\mathbf{Q}_{m}^{\nabla B} L_{m}^{\nabla}\right\|$ by estimating $\Sigma_{m}, \sigma_{m}$, $\rho_{m}$ and $\lambda_{m}$ (see $\left.\S 12\right)$.

\section{EXAMPLES}

We give only three examples, more general examples will be introduced in another paper in order to keep this paper within reasonable bounds.

Example 12.1. We discuss the numerical solution for the dominant equation with constant coefficients

$$
a y(t)+\frac{b}{2 \pi} \int_{0}^{2 \pi} y(\tau) \cot \frac{\tau-t}{2} \mathrm{~d} \tau=f(t), \quad 0 \leq t<2 \pi .
$$

Now $\kappa=0, a-i b=r e^{i \theta}$ where $\theta$ is the characteristic number of (12.1), $w_{1}(t)=$ $w_{2}(t)=1$.

If $[\theta]_{\pi}=\pi / 2$, then this equation becomes (noting $a=0$ and taking $b=1$ )

$$
\frac{1}{2 \pi} \int_{0}^{2 \pi} y(\tau) \cot \frac{\tau-t}{2} \mathrm{~d} \tau=f(t), \quad 0 \leq t<2 \pi .
$$

Under the constraint condition and the unisolving condition

$$
\frac{1}{2 \pi} \int_{0}^{2 \pi} f(\tau) \mathrm{d} \tau=0, \quad \frac{1}{2 \pi} \int_{0}^{2 \pi} y(\tau) \mathrm{d} \tau=N(N \text { is a given constant }),
$$

(12.2) possesses the unique solution

$$
y(t)=-\frac{1}{2 \pi} \int_{0}^{2 \pi} f(\tau) \cot \frac{\tau-t}{2} \mathrm{~d} \tau+N .
$$

We take $\triangle_{n}(t)=\sin \frac{n}{2} t$ and $\nabla_{n}(t)=\cos \frac{n}{2} t$, then $\left(\triangle_{n}, \nabla_{n}\right)$ is a pair of TPs and

$$
\alpha_{n, j}=\frac{2 \pi}{n}, \beta_{n, j}=\frac{(2 j+1) \pi}{n}, u_{n, j}=v_{n, j}=\frac{1}{n}, \quad j=0,1, \cdots, n-1 .
$$

We easily know that the numerical solution and approximate solution of the first kind for SIE (12.2) are

$$
\begin{aligned}
y_{n}\left(\alpha_{n, j}\right) & =\frac{1}{n} \sum_{k=0}^{n-1} f\left(\beta_{n, k}\right) \cot \frac{\alpha_{n, j}-\beta_{n, k}}{2}+N, \quad j=0,1, \cdots, n-1, \\
y_{n}(t) & =f(t) \cot \frac{n}{2} t-\frac{1}{n} \sum_{k=0}^{n-1} f\left(\beta_{n, k}\right) \cot \frac{\beta_{n, k}-t}{2}+N .
\end{aligned}
$$


If $[\theta]_{\pi} \neq \pi / 2$, we rewrite (12.1) for simplicity in the form

$$
\frac{a}{\sqrt{a^{2}+b^{2}}} y(t)+\frac{b}{2 \pi \sqrt{a^{2}+b^{2}}} \int_{0}^{2 \pi} y(\tau) \cot \frac{\tau-t}{2} \mathrm{~d} \tau=\frac{f(t)}{\sqrt{a^{2}+b^{2}}} .
$$

In this case, it possesses the unique solution

$$
y(t)=\frac{a}{a^{2}+b^{2}} f(t)-\frac{b}{2 \pi\left(a^{2}+b^{2}\right)}\left[\int_{0}^{2 \pi} f(\tau) \cot \frac{\tau-t}{2} \mathrm{~d} \tau+\int_{0}^{2 \pi} f(\tau) \mathrm{d} \tau\right] .
$$

We take $\triangle_{n}(t)=\sin \frac{n}{2} t$ and $\nabla_{n}(t)=\sin \left(\frac{n}{2} t-\theta\right)$, then $\left(\triangle_{n}, \nabla_{n}\right)$ is a pair of TPs for SIE (12.9) and

$$
\alpha_{n, j}=\frac{2 j \pi}{n}, \beta_{n, j}=\frac{2\left(j \pi+[\theta]_{\pi}\right)}{n}, u_{n, j}=v_{n, j}=\frac{1}{n}, \quad j=0,1, \cdots, n-1 .
$$

According to the prior proceeding, the numerical solution and the approximate solution of the first kind for SIE (12.8) are, respectively,

$$
y_{n}\left(\alpha_{n, j}\right)=\frac{b}{a^{2}+b^{2}} \sum_{k=0}^{n-1} f\left(\beta_{n, k}\right) \cot \frac{\alpha_{n, j}-\beta_{n, k}}{2}+\frac{b^{2}}{n a\left(a^{2}+^{2}\right)} \sum_{k=0}^{n-1} f\left(\beta_{n, k}\right),
$$

$$
y_{n}(t)=\frac{f(t)}{a+b \cot \frac{n}{2} t}+\frac{b}{n\left(a^{2}+b^{2}\right)}\left[\sum_{k=0}^{n-1} f\left(\beta_{n, k}\right) \cot \frac{t-\beta_{n, k}}{2}+\sum_{k=0}^{n-1} f\left(\beta_{n, k}\right)\right] .
$$

This simple example is just the result given in 16 by Ioakimidis; but he did not discuss the convergence for the approximate solution. Here, noting $\sum_{j=0}^{n-1}\left|u_{n, j}\right|=$ $\sum_{j=0}^{n-1}\left|v_{n, j}\right|=1$ and the foregoing result

$$
\lim _{n \rightarrow \infty}\left\|y-y_{n}\right\|=0 \quad \text { for } \quad f \in C_{2 \pi}^{\prime},
$$

so, the convergence is very clear.

Example 12.2. We still consider $\operatorname{SIE~(12.1).~If~}[\theta]_{\pi}=\pi / 2$, now we take more generally

$$
\triangle_{n}(t)=\sin \left(\frac{n}{2} t+\vartheta\right), \quad \nabla_{n}(t)=\cos \left(\frac{n}{2} t+\vartheta\right),
$$

where $\vartheta$ is an arbitrary real number. Then, $\left(\triangle_{n}, \nabla_{n}\right)$ is a pair of TPs for SIE (12.2) and

$$
\begin{gathered}
\alpha_{n, j}=\frac{2\left(j \pi-[\vartheta]_{\pi}\right)}{n}, \beta_{n, j}=\frac{2\left(j \pi+\pi / 2-[\vartheta]_{\pi}\right)}{n}, u_{n, j}=v_{n, j}=\frac{1}{n}, \\
j=0,1, \cdots, n-1 .
\end{gathered}
$$

Under the constraint condition and the unisolving condition (12.3), the numerical solution is still (12.6) with $\alpha_{n, j}$ and $\beta_{n, j}$ given in (12.15) and the approximate solution of the second kind for SIE (12.2) is, by (11.5)

$$
y_{n}(t)=\sum_{k=0}^{n-1} \Lambda_{n, k}(t) f\left(\beta_{n, k}\right)
$$


with

$$
\Lambda_{n, j}(t)=\left\{\begin{array}{l}
\frac{(-1)^{j}}{n}\left[\cos \left(\frac{n}{2} t+\frac{\pi}{2}+[\vartheta]_{\pi}\right)-\cos \left(\frac{n}{2} \beta_{n, j}+\frac{\pi}{2}+[\vartheta]_{\pi}\right)\right] \cot \frac{t-\beta_{n, j}}{2}, \\
\text { if } n \text { is even, } \\
-\frac{1}{n}\left[\cos \frac{n\left(t-\beta_{n, k}\right)}{2}-\cos \frac{t-\beta_{n, k}}{2}\right] \csc \frac{t-\beta_{n, j}}{2} \\
\text { if } n \text { is odd. }
\end{array}\right.
$$

If $[\theta]_{\pi} \neq \pi / 2$, we take

$$
\triangle_{n}(t)=\sin \left(\frac{n}{2} t+\vartheta\right), \quad \nabla_{n}(t)=\cos \left(\frac{n}{2} t+\vartheta-\theta\right),
$$

then $\left(\triangle_{n}, \nabla_{n}\right)$ is a pair of TPs for SIE (12.8) and

$$
\begin{gathered}
\alpha_{n, j}=\frac{2\left(j \pi-[\vartheta]_{\pi}\right)}{n}, \beta_{n, j}=\frac{2\left(j \pi+\pi / 2-[\vartheta-\theta]_{\pi}\right)}{n}, \\
u_{n, j}=v_{n, j}=\frac{1}{n}, \quad j=0,1, \cdots, n-1 .
\end{gathered}
$$

Now the numerical solution is still (12.11) with $\alpha_{n, j}$ and $\beta_{n, j}$ given in (12.19), and the approximate solution of the second kind for SIE (12.9) is

$$
y_{n}(t)=\sum_{k=0}^{n-1} \Lambda_{n, k}(t) f\left(\beta_{n, k}\right)+\frac{b^{2}}{n a\left(a^{2}+b^{2}\right)} \sum_{k=0}^{n-1} f\left(\beta_{n, k}\right),
$$

where $\Lambda_{n, k}$ is still (12.17) with $\alpha_{n, j}$ and $\beta_{n, j}$ given in (12.19).

In this example, taking $\vartheta=0$ we get the result in [15] by Krenk. He also did not give the convergence for the approximate solution. In fact, the approximate solution of the second kind is convergent in any case, i.e., $\lim _{n \rightarrow \infty}\left\|y-y_{n}\right\|=0$ for $f \in H_{2 \pi}$. To do so, by using the foregoing result in the present paper, it is enough to prove the following lemma.

Lemma 12.1. For any case mentioned above, we have $\rho_{n}=\sum_{j=0}^{n-1}\left\|\Lambda_{n, j}\right\|$ $\leq 2(\pi+\ln n)$.

Proof. For illustration, we consider the case of $\Lambda_{n, j}(t)$ given in (12.17). For fixed $t$, we use the symbol $\tau_{t}$ to denote the congruent point of $\tau$, lying in $[-\pi+t, \pi+t)$. By this view, we rearrange $\left\{\left(\beta_{n, j}\right)_{t}, j=0, \cdots, n-1\right\}$ as $\left\{\beta_{n, k}^{*}, k=1, \cdots, n\right\}$ according to $\beta_{n, 1}^{*}<\beta_{n, 2}^{*}<\cdots<\beta_{n, n}^{*}$. Obviously,

$$
\beta_{n, k+1}^{*}-\beta_{n, k}^{*}=\frac{2 \pi}{n} \text { for } k=1, \cdots, n-1, \quad \beta_{n, \ell}^{*} \leq t<\beta_{n, \ell+1}^{*} \text { for certain } \ell .
$$

Writing

$$
\begin{gathered}
\Lambda_{n, k}^{*}(t)=\Lambda_{n, j}(t) \text { when } \beta_{n, k}^{*}=\left(\beta_{n, j}\right)_{t}, \\
F_{1}(t)=\sum_{k=1}^{\ell-2}\left|\Lambda_{n, k}^{*}(t)\right|, \quad F_{2}(t)=\sum_{k=\ell+3}^{n}\left|\Lambda_{n, k}^{*}(t)\right|,
\end{gathered}
$$


with the assumptions $F_{1}(t)=0$ if $\ell<3$ and $F_{2}(t)=0$ if $\ell>n-3$, we have, for odd $n$,

$$
\begin{aligned}
\left|\Lambda_{n, k}^{*}(t)\right| & =\frac{1}{n}\left|2 \sin \frac{n+1}{4}\left(t-\beta_{n, k}^{*}\right) \sin \frac{n-1}{4}\left(t-\beta_{n, k}^{*}\right) \csc \frac{t-\beta_{n, k}^{*}}{2}\right| \\
& \leq\left|\frac{t-\beta_{n, k}^{*}}{2} \csc \frac{t-\beta_{n, k}^{*}}{2}\right| \leq \frac{\pi}{2} .
\end{aligned}
$$

In the last inequality we used $|\sin x| \geq \frac{2}{\pi}|x|$ for $|x| \leq \frac{\pi}{2}$. Then, also using this inequality and (12.21), we get

$$
\begin{aligned}
F_{1}(t) & =\frac{1}{2 \pi} \sum_{k=1}^{\ell-2}\left|\int_{\beta_{n, k}^{*}}^{\beta_{n, k+1}^{*}}\left[\cos \frac{n\left(t-\beta_{n, k}^{*}\right)}{2}-\cos \frac{t-\beta_{n, k}^{*}}{2}\right] \csc \frac{t-\beta_{n, k}^{*}}{2} \mathrm{~d} x\right| \\
& \leq \frac{1}{\pi} \int_{-\pi+t}^{\beta_{n, \ell-1}^{*}} \csc \frac{t-x}{2} \mathrm{~d} x=-\frac{2}{\pi} \ln \cot \frac{t-t_{\ell-1}^{*}}{4} \\
& \leq-\frac{2}{\pi} \ln \sin \frac{\pi}{2 n} \leq \ln n .
\end{aligned}
$$

Similarly, $\left\|F_{2}\right\| \leq \ln n$. For even $n$, the proof is obtained by working in a similar way.

Example 12.3. We have seen that, in the above examples, the TPs $\left(\triangle_{n}, \nabla_{m}\right)$ all are just some orthogonal polynomials. Now we consider the general SIE (2.8). First, we set up the inner product space $\mathbf{L}_{w_{1}}^{2}[0,2 \pi]$ and $\mathbf{L}_{w_{1}}^{* 2}[0,2 \pi]$, respectively, with the inner product

$$
(f, g)_{1}=\int_{0}^{2 \pi} w_{1}(\tau) f(\tau) g(\tau) \mathrm{d} \tau, \quad(f, g)_{1}^{*}=\int_{0}^{2 \pi} w_{1}(2 \tau) f(\tau) g(\tau) \mathrm{d} \tau .
$$

It is well known that, by applying the Gram-Schmidt orthogonalization process to the system of linear independent polynomials $\{1, \sin \tau, \cos \tau, \cdots, \sin (n-1) \tau, \cos (n-$ 1) $\tau, \sin (n \tau+\vartheta)\}(0 \leq \vartheta<\pi)$, we may get the orthogonal trigonometric polynomial $\Pi_{n}^{\vartheta}(\tau)$ in $\mathbf{L}_{w_{1}}^{* 2}[0,2 \pi]$ (see [34]), i.e.,

$$
\left\{\begin{array}{l}
\Pi_{n}^{\vartheta}(\tau)=c \sin (n \tau+\vartheta)+\text { terms of lower degree, } c \neq 0, \\
\left(\Pi_{n}^{\vartheta}, T_{r}\right)_{1}^{*}=0 \text { for } T_{r} \in H_{n-1}^{T} .
\end{array}\right.
$$

Similarly, we also may get the orthogonal trigonometric polynomial $\triangle_{n}$ in $\mathbf{L}_{w_{1}}^{2}[0,2 \pi]$,

$$
\left\{\begin{array}{l}
\triangle_{n}^{\vartheta}(\tau)=c \sin \left(\frac{n}{2} \tau+\vartheta\right)+\delta_{\frac{n}{2}-1}(\tau), \quad \delta_{\frac{n}{2}-1} \in H_{\frac{n}{2}-1}^{T}, c \neq 0 \\
\left(\triangle_{n}^{\vartheta}, \Upsilon_{r}\right)_{1}=0 \text { for } \Upsilon_{r} \in H_{\frac{n}{2}-1}^{T} .
\end{array}\right.
$$

Remark 12.1. If $c=1$ in (12.25) and (12.26), we say that they are monic. The monic $\Pi_{n}^{\vartheta}$ uniquely exists in each class $H_{n}^{T}(\vartheta)$. The monic $\triangle_{n}^{\vartheta}$ uniquely exists in each class $H_{\frac{n}{2}}^{T}(\vartheta)$. Sometimes we simply write them as $\Pi_{n}$ and $\triangle_{n}$.

Lemma 12.2 (Du [26]). $\Pi_{n}(\tau)=a \prod_{j=1}^{n} \sin \left(\tau-\frac{1}{2} \alpha_{n, j}\right)$ where $a \neq 0$ and $\alpha_{n, j}$ 's are distinct points in $[0,2 \pi)$.

Lemma 12.3. $\triangle_{n}(\tau)=\Pi_{n}\left(\frac{\tau}{2}\right)=a \prod_{j=1}^{n} \sin \frac{\tau-\alpha_{n, j}}{2}$, where $\Pi_{n}$ is as above. 
Proof. When $(n+j)$ is even, for any $\sigma$ we have, by Lemma 12.2,

$$
\int_{0}^{2 \pi} w_{1}(2 \tau) \Pi_{n}(\tau) \sin (j \tau+\sigma) \mathrm{d} \tau=\int_{0}^{2 \pi} w_{1}(\tau) \Pi_{n}\left(\frac{1}{2} \tau\right) \sin \left(\frac{j}{2} \tau+\sigma\right) \mathrm{d} \tau
$$

Thus, the proof is completed by the uniqueness of the monic orthogonal trigonometric polynomial.

Remark 12.2. By changing $w_{1}$ to $w_{2}$, we also introduce the inner product space $\mathbf{L}_{w_{2}}^{2}[0,2 \pi]$ and $\mathbf{L}_{w_{2}}^{* 2}[0,2 \pi]$, then we may obtain similar results. $\nabla \frac{m}{2}$ denotes the orthogonal polynomial in the class $H_{\frac{m}{2}}^{T}(\vartheta)$ on $\mathbf{L}_{w_{2}}^{2}[0,2 \pi]$.

Lemma 12.4. Let $n=2 \ell$ be even, let $\triangle_{n}$ be an orthogonal polynomial in the class $H_{\ell}^{T}(\vartheta)$ on $\mathbf{L}_{w_{1}}^{2}[0,2 \pi]$ and let $\nabla m$ be an orthogonal polynomial in the class $H_{\ell-\kappa}^{T}\left([\vartheta-\theta]_{\pi}\right)$ on $\mathbf{L}_{w_{2}}^{2}[0,2 \pi]$. Then $\mathbf{A} \triangle_{n}=a \nabla_{m}$ and $\mathbf{B} \nabla_{m}=b \triangle_{n}$ where $a$ and $b$ are nonzero constants.

Proof. We only prove the second conclusion. It results directly from (6.2) and (4.8).

Lemma 12.5. For even $n$ large enough, $\mathbf{B} \nabla_{m}=\triangle_{n}$ and $\mathbf{A} \triangle_{n}=\nabla_{m}$ are equivalent.

Proof. $\mathbf{B} \nabla_{m}=\triangle_{n}$ implies $\mathbf{A} \triangle_{n}=\nabla_{m}+b \mathbf{D}_{2} \nabla_{m}=\nabla_{m}$ by Theorem 5.2 and (5.5).

Lemma 12.6. Let $n=2 \ell-1$ be odd, let $\triangle_{n}$ be an orthogonal polynomial in the class $H_{\ell-\frac{1}{2}}^{T}$ on $\mathbf{L}_{w_{1}}^{2}[0,2 \pi]$ and let $\nabla m$ be an orthogonal polynomial in the class $H_{\ell-\kappa-\frac{1}{2}}^{T}$ on $\mathbf{L}_{w_{2}}^{2}[0,2 \pi]$. Then $\overline{\mathbf{A}} \triangle_{n}=a \nabla m$ and $\overline{\mathbf{B}} \nabla_{m}=b \triangle_{n}$ where $a$ and $b$ are nonzero constants.

Proof. Introducing the operator

$$
\left[\overline{\mathbf{A}}^{*} T_{j-\frac{1}{2}}\right](x)=a(x) w_{1}(x) T_{j-\frac{1}{2}}(x)+\frac{1}{2 \pi} \int_{0}^{2 \pi} w_{1}(\tau) b(\tau) T_{j-\frac{1}{2}}(\tau) \csc \frac{\tau-x}{2} \mathrm{~d} \tau,
$$

writing $S_{j}(\tau)=\sin \frac{\tau}{2} T_{j-\frac{1}{2}}(\tau)$ and $C_{j}(\tau)=\cos \frac{\tau}{2} T_{j-\frac{1}{2}}(\tau)$, by (5.10) we have

$$
\begin{aligned}
{\left[\overline{\mathbf{A}}^{*} T_{j-\frac{1}{2}}\right](x)=} & \frac{1}{2 \pi} \int_{0}^{2 \pi} w_{1}(\tau) b(\tau) T_{j-\frac{1}{2}}(\tau) \sin \frac{\tau-x}{2} \mathrm{~d} \tau \\
& +\sin \frac{x}{2}\left[\mathbf{A}^{*} S_{j}\right](x)+\cos \frac{x}{2}\left[\mathbf{A}^{*} C_{j}\right](x),
\end{aligned}
$$

hence, by Theorem 5.4 and Lemma 4.1,

$$
\overline{\mathbf{A}}^{*} T_{j-\frac{1}{2}} \in H_{\max \left\{j-\kappa+\frac{1}{2}, \frac{1}{2}\right\}}^{T} \quad \text { for } \quad T_{j-\frac{1}{2}} \in H_{j-\frac{1}{2}}^{T} .
$$

Again noting

$$
\int_{0}^{2 \pi} w_{1}(\tau)\left(\overline{\mathbf{B}}_{\nabla m}\right)(\tau) T(\tau) \mathrm{d} \tau=\int_{0}^{2 \pi} w_{2}(x) \nabla_{m}(x)\left[\overline{\mathbf{A}}^{*} T\right](x) \mathrm{d} x,
$$

thus, $\overline{\mathbf{B}} \nabla_{m}=b \triangle_{n}$. Similarly, we may prove $\overline{\mathbf{A}} \triangle_{n}=a \nabla_{m}$.

Moreover, we also have

Lemma 12.7. $\overline{\mathbf{B}} \nabla_{m}=\triangle_{n}$ and $\overline{\mathbf{A}} \triangle_{n}=\nabla_{m}$ are equivalent for odd $n$ large enough. 
Summing up the above discussion, we get the main result of this example.

Conclusion. Let $\triangle_{n}$ be an orthogonal polynomial in the class $H_{\frac{n}{2}}^{T}$ on $\mathbf{L}_{w_{1}}^{2}[0,2 \pi]$, take $\nabla_{m}=\mathbf{A} \triangle_{n}$ while $n$ is even and $\nabla_{m}=\overline{\mathbf{A}} \triangle_{n}$ while $n$ is odd. From the above discussion we know that $\left(\triangle_{n}, \nabla_{m}\right)$ is just a pair of TPs for SIE (2.8). Under this scheme, we get the results in [17] and [18] by installing the foregoing proceeding in the present paper to SIE (2.8).

Remark 12.3. In [17, we have proved the convergence of approximate solution of the first kind, because $u_{n, j}$ and $v_{m, k}$ are all positive. The convergence of approximate solution of the second kind depends on the condition $\left\|\mathbf{Q}_{m}^{\nabla B} \mathbf{L}_{m}^{\nabla}\right\|=\mathrm{o}\left(n^{\nu}\right)$, which is now an open problem. We guess that it is true for the present case.

\section{ACKNOWLEDGEMENTS}

The first version of this work was done while the author visited the Free University Berlin in the fall term 2001. The revision of this work was done while the author was visiting the Free University Berlin in the summer term 2005 due to the State Scholarship Fund Award of China. The author is very grateful to Professor Heinrich Begehr for his helpful support.

The author is also very grateful to the referees for several helpful suggestions which led to improvement of this article.

\section{REFERENCES}

[1] E. Venturino, Recent developments in the numerical solution of singular integral equations, J. Math. Anal. Appl., 115(1986), 239-277. MR835600(87i:65213)

[2] Lu Jianke, Du Jinyuan, Numerical methods of solution for singular integral equations, Advances in Mathematics, 20(1991), 3, 278-293.

[3] D. Elliott, The classical collocation method for singular integral equations, SIAM J. Numer. Anal., 19(1982), 816-832. MR664887 (83f:65208)

[4] D. Elliott, Orthogonal polynomials associated with singular integral equations having a Cauchy kernel, SIAM J. Math. Anal., 13(1982), 1041-1052. MR674772 (84a:45003)

[5] D. Elliott, A Galerkin-Petrov method for singular integral equations, J. Austr. Math. Soc. Ser. B, 25(1983), 261-275. MR717483(85h:65272)

[6] D. Elliott, Rates of convergence for the method of classical collocation for solving singular integral equations, SIAM J. Numer. Anal., 21(1984), 136-148. MR731218 (85i:65171)

[7] D. Elliott, A convergence theorem for singular integral equations, J. Austral. Math. Soc. Ser. $B, 22(1981), 539-552$. MR626942 (82m:45020)

[8] D. Elliott, The numerical treatment of singular integral equations - a review, in Treatment of Integral Equations by Numerical Methods, C. T. H. Baker, G. F. Miller (Editors), Acad. Press., New York, 1982. MR0755364

[9] D. Elliott, Singular integral equations on arc $(-1,1)$ : theory and approximate solution, Part 1, Theory, Technical Report No. 218, Dept. of Math., University of Tasmania, Australia, 1987.

[10] D. Elliott, Projection methods for singular integral equations, J. of Integral Equations and Applications, 2(1989), 95-106. MR.1033205 (90m:45007)

[11] Du Jinyuan, Singular integral operators and singular quadrature operators associated with singular integral equations, Acta Math. Sci., 18(1998), 2, 227-240. MR 1645874(2000a:45006)

[12] Du Jinyuan, The classical collocation methods for singular integral equations with Cauchy kernels, Acta Math. Sci., 20(2000), 3, 289-302. MR.1793202 (2001k:65201)

[13] Jian-ke Lu, Boundary Value Problems for Analytic Functions, World Scientific, Singapore, 1993. MR:1279172 (95j:30037)

[14] N. I. Muskhelishvili, Singular Integral Equations, 2nd ed., Noordhoff, Groningen, 1968. MR0355495 (50:7969) 
[15] S. Krenk, Numerical quadrature of periodic singular integral equations, J. Inst. Maths. Applics, 21 (1978), 181-187. MR0483592 (58:3584)

[16] N. I. Ioakimidis, A natural interpolation formula for the numerical solution of singular integral equations with Hilbert kernel, BIT, 23(1983), 92-104. MR689607 (85a:65176)

[17] Du Jinyuan, On the numerical solution for singular integral equations with Hilbert kernel, Chinese J. Num. Math. \& Appl., 11(1989), 2, 9-27.

[18] Du Jinyuan, On the trigonometric polynomials interpolating approximate solutions of singular integral equations with Hilbert kernel, in "Integral Equations and Boundary Value Problems", ed. by G. C. Wen and Z. Zhao, World Scientific, Singapore, 1991, 26-33. MR1111039 (92d:42003)

[19] J. Saranen, G. Vainikko, Periodic Integral and Pseudodifferential Equations with Numerical Approximation, Springer, Berlin, 2002. MR.1870713 (2003h:47088)

[20] J. Saranen, L. Schroderus, The modified quadrature method for classical pseudodifferential equtions of negative order on smooth closed curves, Journal of Computational and Applied Mathematics, 50(1994), 485-495. MR.1284284(95f:65059)

[21] Du Jinyuan, Some systems of orthogonal trigonometric polynomials associated with singular integrals with Hilbert kernel, J. of Wuhan University (Natural Science Edition), 2(1988), 2, 17-35. MR.972923 (90b:45010)

[22] Lu Jianke, On singular integral equations with Hilbert kernel, Chinese Advances in Mathematics, 8(1965), 2, 161-167. MR0212522 (35:3393)

[23] Lu Jianke, General inversion formulas of integrals with Hilbert kernel, J. of Wuhan University, 1(1963), 39-64.

[24] Du Jinyuan and Liu Hua, On convergence of trigometric interpolation for periodic analytic functions, in "Finite or Infinite Dimensional Complex Analysis" (ed. by Joji Kajiwara, Zhong Li and Kwang Ho Shon), Marcel Dekker, Inc., New York, Basel, 2000, 69-76. MR 1771304

[25] Jinyuan Du, Huili Han, Guoxiang Jin, On trigonometric and paratrigonometric Hermite interpolation, J. Approximation Theory, 131(2004), 74-99. MR.2103835(2005i:42005)

[26] Du Jinyuan, Quadrature formulas for singular integrals with Hilbert kernel, J. Comp. Math., 6(1988), 3, 205-225. MR.967882 (90b:41049)

[27] Du Jinyuan, Quadrature formulas of quasi-interpolation type for singular integrals with Hilbert kernel, J. Approximation Theory, 93(1998), 2, 231-257. MR.1616777 (99e:41021)

[28] I. J. Schoenberg, Notes on spline functions, Indagationes Mathematicae, 34(1972), 412-422. MR0316944 (47:5492)

[29] P. Linz, A general theory for the approximate solution of operator equations of the second kind, SIAM J. Numer. Anal., 14(1977), 543-554. MR0431665 (55:4660)

[30] Du Jin Yuan, Numerical solution methods for singular integral equations (II), Acta Math. Sci., 8(1988), 33-45. MR.90a:65271b

[31] I. P. Natanson, Constructive function theory, 3vols, F. Ungar, New York, 1964-1965.

[32] R. A. Devore, G. G. Lorentz, Constructive Approximation, Springer-Verlag, Berlin, Heidelberg, 1993. MR1261635 (95f:41001)

[33] S. Prößdorf and B. Silbermann, Projektionsverfahren und die näherungsweise Lösung singulärer Gleichungen, Teubner-Texte zur Mathematik, Teubner, Leipzig, 1977. MR0494817 $(58: 13602)$

[34] W. Rudin, Functional Analysis, 2nd. ed., World Sci., New York, 1991. MR1157815 (92k:46001)

Department of Mathematics, Wuhan University, Wuhan 430072, People's Republic OF China

E-mail address: jydu@whu.edu.cn 